\title{
Poisson brackets on some skew PBW extensions
}

\author{
B. A. Zambrano \\ Communicated by V. A. Artamonov \\ This article is dedicated to my family
}

\begin{abstract}
A BStRACT. In [1] the author gives a description of Poisson brackets on some algebras of quantum polynomials $\mathcal{O}_{q}$, which is called the general algebra of quantum polynomials. The main of this paper is to present a generalization of [1] through a description of Poisson brackets on some skew PBW extensions of a ring $A$ by the extensions $\mathcal{O}_{q, \delta}^{r, n}$, which are generalization of $\mathcal{O}_{q}$, and show some examples of skew PBW extension where we can apply this description.
\end{abstract}

\section{Introduction}

Skew PBW extensions were introduced in [2] and some of its properties have been studied in [4], [5], [6], [7], among others. Let A and R be rings, we say that $\mathrm{R}$ is a skew PBW extension of $\mathrm{A}$, if the following conditions hold:

1) $A \subset R$

2) There exist finitely many elements $x_{1}, \ldots, x_{n} \in R$ such that $R$ is a left A-free module with basis

$$
\operatorname{Mon}\left\{x_{1}, \ldots, x_{n}\right\}=\left\{x_{1}^{\alpha_{1}} \cdots x_{n}^{\alpha_{n}} \mid\left(\alpha_{1}, \ldots, \alpha_{n}\right) \in \mathbb{N}\right\} .
$$

2010 MSC: 16S10.

Key words and phrases: Poisson brackets, noncommutative rings, skew PBW extensions. 
3) For every $i=1, \ldots, n$ there exist an injective ring endomorphism $\sigma_{i}: A \rightarrow A$ and a $\sigma_{i}$-derivation $\delta_{i}: A \rightarrow A$ such that

$$
x_{i} a=\sigma_{i}(a) x_{i}+\delta_{i}(a)
$$

for all $a \in A$.

4) For every $1 \leqslant i<j \leqslant n$ there exists $q_{i j} \in A$ left invertible and $a_{i j}^{(t)} \in A$ such that

$$
x_{j} x_{i}=q_{i j} x_{i} x_{j}+a_{i j}^{(0)}+a_{i j}^{(1)} x_{1}+\cdots+a_{i j}^{(n)} x_{n}
$$

Under these conditions we will denote $R=\sigma(A)\left\langle x_{1}, \ldots, x_{n}\right\rangle$.

Fix $1 \leqslant r \leqslant n$, we will denote $\mathcal{O}_{q, \delta}^{r, n}$ an extension of A such that

1) $A \subset \mathcal{O}_{q, \delta}^{r, n}$.

2) There exist finitely many elements $x_{1}, \ldots, x_{n}, x_{1}^{-1}, \ldots, x_{r}^{-1} \in \mathcal{O}_{q, \delta}^{r, n}$ such that $\mathcal{O}_{q, \delta}^{r, n}$ is a left A-free module with basis

$$
\operatorname{Mon}\left\{x_{1}^{ \pm}, \ldots, x_{r}^{ \pm}, x_{r+1}, \ldots, x_{n}\right\}=\left\{x_{1}^{\alpha_{1}} \cdots x_{n}^{\alpha_{n}} \mid\left(\alpha_{1}, \ldots, \alpha\right) \in Z\right\},
$$

where $Z=\mathbb{Z}^{r} \times \mathbb{N}^{n-r}$.

3) For every $i=1, \ldots, n$ there exist a derivation $\delta_{i}: A \rightarrow A$ such that

$$
x_{i} a=a x_{i}+\delta_{i}(a)
$$

for all $a \in A$.

4) For every $1 \leqslant i<j \leqslant n$ there exists $q_{i j} \in Z(A)$ invertible and $a_{i j}^{(t)} \in Z(A)$ such that

$$
x_{j} x_{i}=q_{i j} x_{i} x_{j}+a_{i j}^{(0)}+a_{i j}^{(1)} x_{1}+\cdots+a_{i j}^{(n)} x_{n}
$$

5) $x_{i} x_{i}^{-1}=x_{i}^{-1} x_{i}=1$ for $i=1, \ldots, r$.

In the present paper we shall assume that $\mathcal{O}_{q, \delta}^{r, n}$ satisfies the following conditions

1) $\delta_{1}=0$.

2) For every $i$ fixed, $i=1, \ldots, n$ and $\left(m_{1}, \ldots, m_{n}\right) \in Z \backslash\{(0, \ldots, 0)\}$, $\left(1-\prod_{j=1, j \neq i}^{n} q_{i j}^{m_{j}}\right) \in A^{*}$

3) $\delta_{t}\left(q_{i j}\right)=\delta_{t}\left(a_{i j}^{(m)}\right)=0$ for all $m=0, \cdots, n$ and $i, j, t=1, \cdots, n$.

4) $a_{1 j}^{(0)}=0$ for all $j=1, \ldots, n$. If $1 \leqslant i \leqslant r$ and $i<j$ then $a_{i j}^{(m)}=0$ for all $m=i, \cdots, n$. 
We will denote $\bar{p}_{i j}=p_{i j}-a_{i j}^{(0)}$.

Remark 1.1. We will consider the deglex order over $\operatorname{Mon}\left(\mathcal{O}_{q, \delta}^{r, n}\right):=$ $\operatorname{Mon}\left\{x_{1}^{ \pm}, \ldots, x_{r}^{ \pm}, x_{r+1}, \ldots, x_{n}\right\}$ that is defined by [2] as

$$
x^{\alpha} \succeq x^{\beta}= \begin{cases}x^{\alpha}=x^{\beta} & \text { or } \\ x^{\alpha} \neq x^{\beta} \text { but }|\alpha|>|\beta| & \text { or } \\ x^{\alpha} \neq x^{\beta},|\alpha|=|\beta| \text { but } \exists i & \\ \quad \text { with } \alpha_{1}=\beta_{i}, \ldots, \alpha_{i-1}=\beta_{i-1} \text { and } \alpha_{i}>\beta_{i} & \end{cases}
$$

where $x^{\alpha}, x^{\beta} \in \operatorname{Mon}\left(\mathcal{O}_{q, \delta}^{r, n}\right)$. Each element $f \in \mathcal{O}_{q, \delta}^{r, n} \backslash\{0\}$ can be represented in a unique way as $f=\eta_{v_{0}} x^{v_{0}}+\cdots+\eta_{v_{k}} x^{v_{k}}$, with $\eta_{v_{l}} \in A \backslash\{0\}, 1 \leqslant l \leqslant k$, and $x^{v_{k}} \succ \cdots \succ x^{v_{0}}$; we take $\operatorname{deg}(f)=\operatorname{deg}\left(x^{v_{k}}\right):=\left|v_{k}\right|^{1}$. We will denote $i t(f)=\eta_{v_{k}} x^{v_{k}}$ the leader term of $f$.

Lemma 1.2. For all $z \in \mathcal{O}_{q, \delta}^{r, n}, i, j=1, \ldots, n$, and $m=0, \ldots, n$ we have that $q_{i j} z=z q_{i j}$ and $a_{i j}^{(m)} z=z a_{i j}^{(m)}$.

Proof. It is enough to see that $q_{i j} x_{t}=x_{t} q_{i j}$ and $a_{i j}^{(m)} x_{t}=x_{t} a_{i j}^{(m)}$ for all $t$. By the definition of $\mathcal{O}_{q, \delta}^{r, n}$ we have that $x_{t} q_{i j}=q_{i j} x_{t}+\delta_{t}\left(q_{i j}\right)=q_{i j} x_{t}$ and $x_{t} a_{i j}^{(m)}=a_{i j}^{(m)} x_{t}+\delta_{t}\left(a_{i j}^{(m)}\right)=a_{i j}^{(m)} x_{t}$.

Lemma 1.3. Let $x_{1}^{a_{1}} \cdots x_{n}^{a_{n}} \in \operatorname{Mon}\left(\mathcal{O}_{q, \delta}^{r, n}\right)$ then

1) For all $i=1, \ldots, n$ there exists $p_{i, a} \in \mathcal{O}_{q, \delta}^{r, n}$ such that $x_{1}^{a_{1}} \cdots x_{n}^{a_{n}} x_{i}=$ $\left(\prod_{j>i} q_{i j}^{a_{j}}\right) x_{1}^{a_{1}} \cdots x_{i}^{a_{i}+1} \cdots x_{n}^{a_{n}}+p_{i, a}$ with $\operatorname{deg}\left(p_{i, a}\right)<a_{1}+\cdots+a_{n}+1$ or $p_{i, a}=0$ where $a=\left(a_{1}, \ldots, a_{n}\right)$.

2) For all $i=1, \ldots, n$ there exists $p_{a, i} \in \mathcal{O}_{q, \delta}^{r, n}$ such that $x_{i} x_{1}^{a_{1}} \cdots x_{n}^{a_{n}}=$ $\left(\prod_{j<i} q_{j i}^{a_{j}}\right) x_{1}^{a_{1}} \cdots x_{i}^{a_{i}+1} \cdots x_{n}^{a_{n}}+p_{a, i}$ with $\operatorname{deg}\left(p_{a, i}\right)<a_{1}+\cdots+a_{n}+1$ or $p_{a, i}=0$ where $a=\left(a_{1}, \ldots, a_{n}\right)$.

Proof. Fix $x_{i} \in \mathcal{O}_{q, \delta}^{r, n}$ and take $x_{t_{1}}^{a_{1}} \cdots x_{t_{j}}^{a_{j}} \in \mathcal{O}_{q, \delta}^{r, n}$ such that $a_{l} \neq 0$. We will prove the first claimed by induction on $j$.

1) $\left(j=1, x_{t_{1}}^{a_{1}}=x_{t}^{b}\right)$ We will show this claimed by induction on $b$, we can suppose that $i<t$, in other way, we have the claimed.

(a) $(b=1)$ By definition, for all $i<t$ we have that $x_{t} x_{i}=q_{i t} x_{i} x_{t}+p_{i, t}$, where $\operatorname{deg}\left(p_{i, t}\right)<2$.

${ }^{1}$ If $\alpha=\left(\alpha_{1}, \ldots, \alpha_{n}\right) \in \mathbb{Z}^{n}$ then we take $|\alpha|=\alpha_{1}+\cdots+\alpha_{n}$. 
(b) $(b+1)$ Put $i<t$. By induction hypothesis there exist $p_{l, b}$ such that $x_{t}^{b} x_{l}=q_{l t}^{b} x_{l} x_{t}^{b}+p_{l, b}$ where $\operatorname{deg}\left(p_{l, b}\right)<b+1$ for all $l<t$, then

$$
\begin{aligned}
x_{t}^{b+1} x_{i}= & x_{t}^{b}\left(q_{i t} x_{i} x_{t}+a_{i t}^{(0)}+\sum_{l=1}^{n} a_{i t}^{(l)} x_{l}\right) \\
= & q_{i t} x_{t}^{b} x_{i} x_{t}+\left(a_{i t}^{(0)} x_{t}^{b}+\sum_{l=1}^{n} a_{i t}^{(l)} x_{t}^{b} x_{l}\right) \\
= & q_{i t} q_{i t}^{b} x_{i} x_{t}^{b+1}+q_{i t} p_{i, b}+a_{i t}^{(0)} x_{t}^{b}+\sum_{l=1}^{t-1} a_{i t}^{(l)} q_{l t}^{b} x_{l} x_{t}^{b} \\
& +\sum_{l=1}^{t-1} a_{i t}^{(l)} p_{l, b}+\sum_{l=t}^{n} a_{i t}^{(l)} x_{t}^{b} x_{l} \\
= & q_{i t}^{b+1} x_{i} x_{t}^{b+1}+p_{i, b+1}
\end{aligned}
$$

where $p_{i, b+1}=a_{i t}^{(0)} x_{t}^{b}+\sum_{l=1}^{t-1} a_{i t}^{(l)} q_{l t}^{b} x_{l} x_{t}^{b}+\sum_{l=1}^{t-1} a_{i t}^{(l)} p_{l, b}+\sum_{l=t}^{n} a_{i t}^{(l)} x_{t}^{b} x_{l}$ and

$$
\operatorname{deg}\left(a_{i t}^{(0)} x_{t}^{b}\right), \operatorname{deg}\left(a_{i t}^{(l)} q_{l t}^{b} x_{l} x_{t}^{b}\right), \operatorname{deg}\left(a_{i t}^{(l)} p_{l, b}\right), \operatorname{deg}\left(a_{i t}^{(l)} x_{t}^{b} x_{l}\right)<b+2
$$

(c) $(b=-1$, if $t \leqslant r)$ We will show that $p_{i,-1}=\sum_{h=1}^{i-1} \sum_{k=1}^{s(i,-1)} r_{h, k} x_{h} x_{t}^{-k}$ with $r_{h, k} \in\left\langle a_{i j}^{(m)}, q_{i j} \mid i, j=1, \ldots, n, m=0, \ldots, n\right\rangle=G$, sub-ring of $A$, by induction on $1 \leqslant i \leqslant r$. If $i=1$ then $x_{t}^{-1} x_{1}=x_{t}^{-1}\left(x_{1} x_{t}\right) x_{t}^{-1}=$ $x_{t}^{-1}\left(q_{t 1} x_{t} x_{1}\right) x_{t}^{-1}=q_{1 t}^{-1} x_{1} x_{t}^{-1}$ and $p_{1,-t}=0$. If $i>1$ by induction hypothesis we have that

$$
\begin{aligned}
x_{t}^{-1} x_{i} & =x_{t}^{-1}\left(x_{i} x_{t}\right) x_{t}^{-1}=x_{t}^{-1}\left(q_{t i} x_{t} x_{i}+a_{t i}^{(0)}+\sum_{l=1}^{i-1} a_{t i}^{(l)} x_{l}\right) x_{t}^{-1} \\
& =q_{t i} x_{i} x_{t}^{-1}+a_{t i}^{(0)} x_{t}^{-2}+\sum_{l=1}^{i-1} a_{t i}^{(l)} x_{t}^{-1} x_{l} x_{t}^{-1} \\
& =q_{i t}^{-1} x_{i} x_{t}^{-1}+a_{t i}^{(0)} x_{t}^{-2}+\sum_{l=1}^{i-1} a_{t i}^{(l)} q_{l t}^{-1} x_{l} x_{t}^{-2}+\sum_{l=1}^{i-1} a_{t i}^{(l)} p_{l,-t} x_{t}^{-1}
\end{aligned}
$$

since $p_{l,-t}=\sum_{h=1}^{l-1} \sum_{k=1}^{s(l,-1)} r_{h, k} x_{h} x_{t}^{-k}$ with $\operatorname{deg}\left(p_{l,-t}\right)<0$ for all $l=$ $1, \ldots, i-1$ then $\operatorname{deg}\left(p_{l,-t}\right) x_{t}^{-1}<-1$ for all $l$ and

$$
p_{l,-t} x_{t}^{-1}=\sum_{h=1}^{l-1} \sum_{k=1}^{s(l,-1)} r_{h, k} x_{h} x_{t}^{-k-1}
$$


so we have the claimed.

(d) $(-(b+1), b>0$ if $t \leqslant r)$ The same way, put $i<t$, by induction hypothesis there exist $p_{i,-1}, p_{i,-b}$ as in (c) such that $x_{t}^{-1} x_{i}=q_{i t}^{-1} x_{i} x_{t}^{-1}+$ $p_{i,-1}$ and $x_{t}^{-b} x_{i}=q_{i,-t}^{-b} x_{i} x_{t}^{-b}+p_{i,-b}$, with $\operatorname{deg}\left(p_{i,-1}\right)<0$ and $\operatorname{deg}\left(p_{i,-b}\right)<$ $-b+1$ then

$$
\begin{aligned}
x_{t}^{-b-1} x_{i} & =x_{t}^{-1}\left(x_{t}^{-b} x_{i}\right)=x_{t}^{-1}\left(q_{i t}^{-b} x_{i} x_{t}^{-b}+p_{i,-b}\right) \\
& =q_{i t}^{-b} x_{t}^{-1} x_{i} x_{t}^{-b}+x_{t}^{-1} p_{i,-b}=q_{i t}^{-b}\left(q_{i t}^{-1} x_{i} x_{t}^{-1}+b_{i,-1}\right) x_{t}^{-b}+x_{t}^{-1} p_{i,-b} \\
& =q_{i t}^{-b-1} x_{i} x_{t}^{-b-1}+q_{i t}^{-b} b_{i,-1} x_{t}^{-b}+x_{t}^{-1} \sum_{h=1}^{i-1} \sum_{k=1}^{s(i,-b)} r_{h, k} x_{h} x_{t}^{-k} \\
& =q_{i t}^{-b-1} x_{i} x_{t}^{-b-1}+q_{i t}^{-b} b_{i,-1} x_{t}^{-b}+\sum_{h=1}^{i-1} \sum_{k=1}^{s(i,-b)} r_{h, k} x_{t}^{-1} x_{h} x_{t}^{-k} \\
& =q_{i t}^{-b-1} x_{i} x_{t}^{-b-1}+q_{i t}^{-b} b_{i,-1} x_{t}^{-b}+\sum_{h=1}^{i-1} \sum_{k=1}^{s(i,-b)} r_{h, k} q_{h t}^{-1} x_{h} x_{t}^{-b-1} \\
& \quad+\sum_{h=1}^{i-1} \sum_{k=1}^{s(i,-b)} r_{h, k} p_{h,-1} x_{t}^{-b}
\end{aligned}
$$

Note that $x_{t} r_{h, k}=r_{h, k} x_{t}$ for all $h, k$ because for all $t$ and $g \in G, \delta_{t}(g)=0$ so

$$
p_{i,-b-1}=b_{i,-1} x_{t}^{-b}+\sum_{h=1}^{i-1} \sum_{k=1}^{s(i,-b)} r_{h, k} q_{h t}^{-1} x_{h} x_{t}^{-b-1}+\sum_{h=1}^{i-1} \sum_{k=1}^{s(i,-b)} r_{h, k} p_{h,-1} x_{t}^{-b}
$$

where we have the claimed.

2) $(j+1)$ We can suppose that $t_{i}=i$ and $i<t_{j+1}$ because in other way we can put $x_{i}^{a_{i}}$ with $a_{i}=0$. By induction hypothesis on $j=1$ there exist $p_{i, t_{j+1}}$ such that $\operatorname{deg}\left(p_{i, t_{j+1}}\right)<a_{j+1}+1$ and $x_{t_{j+1}}^{a_{j+1}} x_{i}=$ $q_{i t_{j+1}}^{a_{j+1}} x_{i} x_{t_{j+1}}^{a_{j+1}}+p_{i, t_{j+1}}$, again by induction hypothesis there exist $p_{i, t^{\prime}}, t^{\prime}=$ $\left(t_{1}, \ldots, t_{j}\right)$, such that $\operatorname{deg}\left(p_{i, t^{\prime}}\right)<a_{1}+\cdots+a_{i}+1$ and $x_{t_{1}}^{a_{1}} \cdots x_{t_{i}}^{a_{i}} \cdots x_{t_{j}}^{a_{j}} x_{i}=$ $\left(\prod_{l=i+1}^{j} q_{i t_{l}}^{a_{l}}\right) x_{t_{1}}^{a_{1}} \cdots x_{t_{i}}^{a_{i}+1} \cdots x_{t_{j}}^{a_{j}}+p_{i, t^{\prime}}$ then

$$
\begin{aligned}
x_{t_{1}}^{a_{1}} & \cdots x_{t_{j}}^{a_{j}} x_{t_{j+1}}^{a_{j+1}} x_{i}=x_{t_{1}}^{a_{1}} \cdots x_{t_{j}}^{a_{j}}\left(q_{i t_{j+1}}^{a_{j+1}} x_{i} x_{t_{j+1}}^{a_{j+1}}+p_{i, t_{j+1}}\right) \\
& =\left(x_{t_{1}}^{a_{1}} \cdots x_{t_{j}}^{a_{j}} q_{i t_{j+1}}^{a_{j+1}} x_{i} x_{t_{j+1}}^{a_{j+1}}\right)+\left(x_{t_{1}}^{a_{1}} \cdots x_{t_{j}}^{a_{j}} p_{i, t_{j+1}}\right) \\
& =q_{i t_{j+1}}^{a_{j+1}}\left(x_{t_{1}}^{a_{1}} \cdots x_{t_{j}}^{a_{j}} x_{i} x_{t_{j+1}}^{a_{j+1}}\right)+\left(x_{t_{1}}^{a_{1}} \cdots x_{t_{j}}^{a_{j}} p_{i, t_{j+1}}\right)
\end{aligned}
$$




$$
\begin{aligned}
= & q_{i t_{j+1}}^{a_{j+1}}\left(\left(\prod_{l=i+1}^{j} q_{i t_{l}}^{a_{l}}\right) x_{t_{1}}^{a_{1}} \cdots x_{t_{i}}^{a_{i}+1} \cdots x_{t_{j}}^{a_{j}}+p_{i, t^{\prime}}\right) x_{t_{j+1}}^{a_{j+1}} \\
& +\left(x_{t_{1}}^{a_{1}} \cdots x_{t_{j}}^{a_{j}} p_{i, t_{j+1}}\right) \\
= & \left(\prod_{l=i+1}^{j+1} q_{i t_{l}}^{a_{l}}\right) x_{t_{1}}^{a_{1}} \cdots x_{t_{i}}^{a_{i}+1} \cdots x_{t_{j+1}}^{a_{j+1}}+q_{i t_{j+1}}^{a_{j+1}} p_{i, t^{\prime}} x_{t_{j+1}}^{a_{j+1}}+x_{t_{1}}^{a_{1}} \cdots x_{t_{j}}^{a_{j}} p_{i, t_{j+1}} \\
= & \left(\prod_{l=i+1}^{j+1} q_{i t_{l}}^{a_{l}}\right) x_{t_{1}}^{a_{1}} \cdots x_{t_{i}}^{a_{i}+1} \cdots x_{t_{j+1}}^{a_{j+1}}+p_{i, t}
\end{aligned}
$$

where $\operatorname{deg}\left(q_{i t_{j+1}}^{a_{j+1}} p_{i, t^{\prime}} x_{t_{j+1}}^{a_{j+1}}\right) \leqslant \operatorname{deg}\left(p_{i, t^{\prime}}\right)+\operatorname{deg}\left(x_{t_{j+1}}^{a_{j+1}}\right)<a_{1}+\cdots+a_{j}+1+a_{j+1}$ and $\operatorname{deg}\left(x_{t_{1}}^{a_{1}} \cdots x_{t_{j}}^{a_{j}} p_{i, t_{j+1}}\right) \leqslant \operatorname{deg}\left(x_{t_{1}}^{a_{1}} \cdots x_{t_{j}}^{a_{j}}\right)+\operatorname{deg}\left(p_{i, t_{j+1}}\right)<a_{1}+\cdots+$ $a_{j}+a_{j+1}+1$ later $\operatorname{deg}\left(p_{i t}\right)=\operatorname{deg}\left(q_{i t_{j+1}}^{a_{j+1}} p_{i, t^{\prime}} x_{t_{j+1}}^{a_{j+1}}+x_{t_{1}}^{a_{1}} \cdots x_{t_{j}}^{a_{j}} p_{i, t_{j+1}}\right)<$ $a_{1}+\cdots+a_{j+1}+1$. The second claimed is proved the same way we proved the first claimed.

\section{Derivations over $\mathcal{O}_{q, \delta}^{r, n}$}

Definition 2.1. Let $d$ be a linear operator over $\mathcal{O}_{q, \delta}^{r, n}$ such that

$$
d(a b)=d(a) b+\gamma(a) d(b)+\sum_{s} \theta_{s} \alpha_{s}(a) \beta_{s}(b)
$$

for all $a, b \in \mathcal{O}_{q, \delta}^{r, n}$, where $\theta_{s} \in A$ and $\gamma, \alpha_{s}, \beta_{s}$ are toric automorphisms of $A$, i.e. $\gamma\left(x_{i}\right)=\gamma_{i} x_{i}, \alpha_{s}\left(x_{i}\right)=a_{s, i} x_{i}$, and $\beta_{s}\left(x_{i}\right)=b_{s, i} x_{i}$ for all $i=1, \ldots, n$ where $\gamma_{i}, a_{s, i}, b_{s, i} \in A$.

1) If $\theta_{s}=0$ for all $s$ then $d$ is a $\gamma$-derivation.

2) If $\theta_{s}=0$ and $\gamma$ is the identity of $A$ then $d$ is a derivation.

3) A inner $\gamma$-derivation $\left[\operatorname{ad}_{\gamma} u\right] a$ is defined by

$$
\left[\operatorname{ad}_{\gamma} u\right] a:=u a-\gamma(a) u
$$

when $\gamma$ is the identity we denote $\left[\operatorname{ad}_{\gamma} u\right] a=[\operatorname{ad} u] a$.

Lemma 2.2. Let $u_{i}=d\left(x_{i}\right)$ then they are a solution of

$$
\begin{aligned}
u_{j} x_{i} & +\gamma\left(x_{j}\right) u_{i}-q_{i j} u_{i} x_{j}-q_{i j} \gamma\left(x_{i}\right) u_{j}+\theta_{i j} x_{i} x_{j}+K x_{i} \\
& +K^{\prime} x_{j}+\hat{\theta}_{i j} p_{i j}\left(x_{1}, \ldots, x_{n}\right)-\bar{p}_{i j}\left(u_{1}, \ldots, u_{n}\right)+a_{i j}^{(0)} \theta=0
\end{aligned}
$$

where $\hat{\theta}_{i j}, \theta_{i j}, K, K^{\prime} \in A, K=0$ if $\delta_{j}=0$, and $K^{\prime}=0$ if $\delta_{i}=0$. 
Proof. Note that $d(1)=d(1 \cdot 1)=d(1)+d(1)+\sum_{s} \theta_{s}=2 d(1)+\theta$, so we have that

$$
\begin{aligned}
d\left(x_{j} x_{i}\right)= & d\left(q_{i j} x_{i} x_{j}+p_{i j}\left(x_{1}, \ldots, x_{n}\right)\right)=q_{i j} d\left(x_{i}\right) x_{j}+q_{i j} \gamma\left(x_{i}\right) d\left(x_{j}\right) \\
& +q_{i j} \sum_{s} \theta_{s} a_{s, i} x_{i} b_{s, j} x_{j}+d\left(a_{i j}^{(0)}\right)+d\left(a_{i j}^{(1)} x_{1}\right)+\cdots+d\left(a_{i j}^{(n)} x_{n}\right) \\
= & q_{i j} d\left(x_{i}\right) x_{j}+q_{i j} \gamma\left(x_{i}\right) d\left(x_{j}\right)+K_{1} x_{i} x_{j} \\
\quad & \quad \sum_{s} \theta_{s} a_{s, i} \delta_{i}\left(b_{s, i}\right) x_{j}+\bar{p}_{i j}\left(u_{1}, \ldots, u_{n}\right)+a_{i j}^{(0)} d(1) \\
= & q_{i j} u_{i} x_{j}+q_{i j} \gamma\left(x_{i}\right) u_{j}+K_{1} x_{i} x_{j}+K_{2} x_{j}+\bar{p}_{i j}\left(u_{1}, \ldots, u_{n}\right)-a_{i j}^{(0)} \theta .
\end{aligned}
$$

On the other hand,

$$
\begin{aligned}
d\left(x_{j} x_{i}\right) & =u_{j} x_{i}+\gamma\left(x_{j}\right) u_{i}+\sum_{s} \theta_{s} a_{s, j} x_{j} b_{s, i} x_{i} \\
& =u_{j} x_{i}+\gamma\left(x_{j}\right) u_{i}+\sum_{s} \theta_{s} a_{s, j} b_{s, i} x_{j} x_{i}+\sum_{s} \theta_{s} a_{s, j} \delta_{j}\left(b_{s, i}\right) x_{i} \\
& =u_{j} x_{i}+\gamma\left(x_{j}\right) u_{i}+K_{3} x_{i}+K_{4}\left(q_{i j} x_{i} x_{j}+p_{i j}\left(x_{1}, \ldots, x_{n}\right)\right)
\end{aligned}
$$

so that (1) holds.

Lemma 2.3. Let $z \in \mathcal{O}_{q, \delta}^{r, n}$ then $u_{i}-\left[\operatorname{ad}_{\gamma} z\right] x_{i}$ are solutions of (1).

Proof.

$$
\begin{aligned}
\left(u_{j}-\right. & {\left.\left[\operatorname{ad}_{\gamma} z\right] x_{j}\right) x_{i}+\gamma\left(x_{j}\right)\left(u_{i}-\left[\operatorname{ad}_{\gamma} z\right] x_{i}\right)-q_{i j}\left(u_{i}-\left[\operatorname{ad}_{\gamma} z\right] x_{i}\right) x_{j} } \\
& -q_{i j} \gamma\left(x_{i}\right)\left(u_{j}-\left[\operatorname{ad}_{\gamma} z\right] x_{j}\right)+\theta_{i j} x_{i} x_{j}+K x_{i}+K^{\prime} x_{j} \\
& +\hat{\theta}_{i j} p_{i j}\left(x_{1}, \ldots, x_{n}\right)-\bar{p}_{i j}\left(\left(u_{1}-\left[\operatorname{ad}_{\gamma} z\right] x_{1}\right), \ldots,\left(u_{n}-\left[\operatorname{ad}_{\gamma} z\right] x_{n}\right)\right) \\
& +a_{i j}^{(0)} \theta \\
= & \left(u_{j} x_{i}+\gamma\left(x_{j}\right) u_{i}-q_{i j} u_{i} x_{j}-q_{i j} \gamma\left(x_{i}\right) u_{j}+\theta_{i j} x_{i} x_{j}+K x_{i}+K^{\prime} x_{j}\right. \\
& \left.+\hat{\theta}_{i j} p_{i j}\left(x_{1}, \ldots, x_{n}\right)+a_{i j}^{(0)} \theta\right)+\left(-\left[\operatorname{ad}_{\gamma} z\right] x_{j}\right) x_{i}-\gamma\left(x_{j}\right)\left[\operatorname{ad}_{\gamma} z\right] x_{i} \\
& +q_{i j}\left(\left[\operatorname{ad}_{\gamma} z\right] x_{i}\right) x_{j}+q_{i j} \gamma\left(x_{i}\right)\left[\operatorname{ad}_{\gamma} z\right] x_{j}-\sum_{t=1}^{n} a_{i j}^{(t)}\left(u_{t}-\left[\operatorname{ad}_{\gamma} z\right] x_{t}\right) \\
= & \left(u_{j} x_{i}+\gamma\left(x_{j}\right) u_{i}-q_{i j} u_{i} x_{j}-q_{i j} \gamma\left(x_{i}\right) u_{j}+\theta_{i j} x_{i} x_{j}+K x_{i}+K^{\prime} x_{j}\right. \\
& \left.+\hat{\theta}_{i j} p_{i j}\left(x_{1}, \ldots, x_{n}\right)-\bar{p}_{i j}\left(u_{1}, \ldots, u_{n}\right)+a_{i j}^{(0)} \theta\right)+\left(-\left[\operatorname{ad}_{\gamma} z\right] x_{j}\right) x_{i} \\
& -\gamma\left(x_{j}\right)\left[\operatorname{ad}_{\gamma} z\right] x_{i}+q_{i j}\left(\left[\operatorname{ad}_{\gamma} z\right] x_{i}\right) x_{j}+q_{i j} \gamma\left(x_{i}\right)\left[\operatorname{ad}_{\gamma} z\right] x_{j}
\end{aligned}
$$




$$
\begin{aligned}
& +\sum_{t=1}^{n} a_{i j}^{(t)}\left(\left[\mathrm{ad}_{\gamma} z\right] x_{t}\right) \\
= & -z x_{j} x_{i}+\gamma\left(x_{j}\right) z x_{i}-\gamma\left(x_{j}\right) z x_{i}+\gamma\left(x_{j}\right) \gamma\left(x_{i}\right) z+q_{i j} z x_{i} x_{j} \\
& -q_{i j} \gamma\left(x_{i}\right) z x_{j}+q_{i j} \gamma\left(x_{i}\right) z x_{j}-q_{i j} \gamma\left(x_{i}\right) \gamma\left(x_{j}\right) z \\
& +\sum_{t=1}^{n} a_{i j}^{(t)}\left(z x_{t}-\gamma\left(x_{t}\right) z\right) \\
= & {\left[-z x_{j} x_{i}+q_{i j} z x_{i} x_{j}+\sum_{t=1}^{n} a_{i j}^{(t)} z x_{t}+a_{i j}^{(0)} z\right]+\left[\gamma\left(x_{j}\right) z x_{i}-\gamma\left(x_{j}\right) z x_{i}\right] } \\
& +\left[-q_{i j} \gamma\left(x_{i}\right) z x_{j}+q_{i j} \gamma\left(x_{i}\right) z x_{j}\right]+\left[\gamma\left(x_{j}\right) \gamma\left(x_{i}\right) z-\gamma\left(x_{j}\right) \gamma\left(x_{i}\right) z\right] \\
& +\left[\gamma\left(x_{j}\right) \gamma\left(x_{i}\right) z-q_{i j} \gamma\left(x_{i} x_{j}\right) z-\sum_{t=1}^{n} a_{i j}^{(t)} \gamma\left(x_{t}\right) z-a_{i j}^{(0)} z\right] \\
= & {\left[z\left(-x_{j} x_{i}+q_{i j} x_{i} x_{j}+\sum_{t=1}^{n} a_{i j}^{(t)} x_{t}+a_{i j}^{(0)}\right)\right] } \\
& +\left[\gamma\left(x_{j} x_{i}-q_{i j} x_{i} x_{j}-\sum_{t=1}^{n} a_{i j}^{(t)} x_{i}-a_{i j}^{(0)}\right) z\right]=0 .
\end{aligned}
$$

Proposition 2.4. Let $v=a x_{1}^{m_{1}} \cdots x_{n}^{m_{n}}$ with $a \in A$ and $m=\left(m_{1}, \ldots\right.$, $\left.m_{n}\right) \in Z$ such that either $\gamma_{1} \neq 1$ and $\left(1-\gamma_{1} \prod_{j=2}^{n} q_{j 1}^{m_{j}}\right) \in A^{*}$ or $\gamma_{1}=1$ and exists $m_{j} \neq 0$ for $j \neq 1$. Then there exists $w \in A^{*}$ with

$$
\left[\operatorname{ad}_{\gamma} w v x_{1}^{-1}\right] x_{1}=v
$$

Proof. Take $w=\left(1-\gamma_{1} \prod_{j=2}^{n} q_{j 1}^{m_{j}}\right)^{-1}$ that exists by hypothesis. Note that $\gamma_{1} w=w \gamma_{1}$ so

$$
\begin{aligned}
{\left[\operatorname{ad}_{\gamma}\right.} & \left.w v x_{1}^{-1}\right] x_{1}=w v x_{1}^{-1} x_{1}-\gamma_{1} x_{1} w v x_{1}^{-1} \\
& =w\left(v-\gamma_{1} a x_{1} x_{1}^{m_{1}} \cdots x_{n}^{m_{n}} x_{1}^{-1}\right) \\
& =w\left(v-\gamma_{1} a x_{1}^{m_{1}} x_{1} x_{2}^{m_{2}} \cdots x_{n}^{m_{n}} x_{1}^{-1}\right) \\
& =w\left(v-\gamma_{1} a x_{1}^{m_{1}} q_{21}^{m_{2}} x_{2}^{m_{2}} x_{1} \cdots x_{n}^{m_{n}} x_{1}^{-1}\right) \\
& =w\left(v-\gamma_{1} a x_{1}^{m_{1}} q_{21}^{m_{2}} x_{2}^{m_{2}} q_{31}^{m_{3}} x_{3} \cdots q_{n 1}^{m_{n}} x_{n}^{m_{n}} x_{1} x_{1}^{-1}\right) \\
& =w\left(v-\gamma_{1} \prod_{j=2}^{n} q_{j 1}^{m_{j}} a x_{1}^{m_{1}} \cdots x_{n}^{m_{n}}\right)=w\left(1-\gamma_{1} \prod_{j=2}^{n} q_{j 1}^{m_{j}}\right) v=v .
\end{aligned}
$$

Corollary 2.5. Let $v=\sum_{t} \rho_{t} X_{t} \in \mathcal{O}_{q, \delta}^{r, n}$ such that $\rho_{t} X_{t}$ satisfies the hypothesis of the above proposition for all $t$, where $X_{t} \in \operatorname{Mon}\left(\mathcal{O}_{q, \delta}^{r, n}\right)$. Then 
there exists $w \in \mathcal{O}_{q, \delta}^{r, n}$ such that

$$
\left[\operatorname{ad}_{\gamma} w\right] x_{1}=v
$$

Proof. We have that $\left[\operatorname{ad}_{\gamma} w+w^{\prime}\right] z=\left(w+w^{\prime}\right) z-\gamma(z)\left(w+w^{\prime}\right)=(w z-$ $\gamma(z) w)+\left(w^{\prime} z-\gamma(z) w\right)=\left[\operatorname{ad}_{\gamma} w\right] z+\left[\operatorname{ad}_{\gamma} w^{\prime}\right] z$ for all $w, w^{\prime}, z \in \mathcal{O}_{q, \delta}^{r, n}$ so for each $\rho_{t} X_{t}$ there exists $w_{t} \in A$ such that

$$
\left[\operatorname{ad}_{\gamma} w_{t} \rho_{t} X_{t} x_{1}^{-1}\right] x_{1}=\rho_{t} X_{t}
$$

then

$$
\begin{aligned}
v & =\sum_{t} \rho_{t} X_{t}=\sum_{t}\left[\operatorname{ad}_{\gamma} w_{t} \rho_{t} X_{t} x_{1}^{-1}\right] x_{1}=\left[\operatorname{ad}_{\gamma} \sum_{t} w_{t} \rho_{t} X_{t} x_{1}^{-1}\right] x_{1} \\
& =\left[\operatorname{ad}_{\gamma} w\right] x_{1} .
\end{aligned}
$$

Theorem 2.6. Let $u_{i}=d\left(x_{i}\right) \in \mathcal{O}_{q, \delta}^{r, n}$ for $i=1, \ldots, n$.

1) If $\gamma_{1} \neq 1$ and $\left(\prod_{j=2}^{n} q_{j 1}^{m_{j}}-\gamma_{1} q_{1 t}\right) \in A^{*}$ for all $\left(0, m_{2}, \ldots, m_{n}\right) \in Z$. Then there exists $w \in \mathcal{O}_{q, \delta}^{r, n}$ and $\rho_{j} \in A$ such that

$$
u_{1}=\left[\operatorname{ad}_{\gamma} w\right] x_{1} \quad \text { and } \quad u_{j}=\rho_{j} x_{j}+\left[\operatorname{ad}_{\gamma} w\right] x_{j}
$$

for all $j \neq 1$ where $\left(q_{1 t}-q_{1 t} \gamma_{1}\right) \rho_{t}=-\theta_{1 t}$.

2) If $\gamma_{j}=1$ for all $i=1, \ldots, n$. Then there exists $w \in \mathcal{O}_{q, \delta}^{r, n}$ such that

$$
u_{j}=\lambda_{j} x_{j}+[\operatorname{ad} w] x_{j}
$$

for all $j=1, \ldots, n$ and some $\lambda_{j}=\lambda\left(x_{j}\right) \in A$.

Proof. 1) By the above corollary there exists $w \in \mathcal{O}_{q, \delta}^{r, n}$ such that $u_{1}=$ $\left[\operatorname{ad}_{\gamma} w\right] x_{1}$ so we $u_{1}=\left[\operatorname{ad}_{\gamma} w\right] x_{1}$ so we define $\bar{u}_{t}=u_{t}-\left[\operatorname{ad}_{\gamma} w\right] x_{t}$ where they hold the equation (1). In

$$
0=\bar{u}_{t} x_{1}-q_{1 t} \gamma_{1} x_{1} \bar{u}_{t}+\theta_{1 t} x_{1} x_{t}+K_{t} x_{1}
$$

If $\bar{u}_{t}=\sum_{m \in Z} \eta_{m} x_{1}^{m_{1}} \cdots x_{n}^{m_{n}} \in \mathcal{O}_{q, \delta}^{r, n}$ we have

$$
\begin{aligned}
0 & =\left(\sum_{m \in Z} \eta_{m} x_{1}^{m_{1}} \cdots x_{n}^{m_{n}} x_{1}-\gamma_{1} q_{1 t} \sum_{m \in Z} \eta_{m} x_{1} x_{1}^{m_{1}} \cdots x_{n}^{m_{n}}\right) \\
& +\theta_{1 t} x_{1} x_{t}+K x_{t} \\
& =\sum_{m \in Z} \eta_{m} \prod_{j=2}^{n} q_{1 j}^{m_{j}} x_{1} x_{1}^{m_{1}} \cdots x_{n}^{m_{n}}-\gamma_{1} q_{1 t} \sum_{m \in Z} \eta_{m} x_{1} x_{1}^{m_{1}} \cdots x_{n}^{m_{n}}
\end{aligned}
$$




$$
\begin{aligned}
& +\theta_{1 t} x_{1} x_{t}+K x_{t} \\
& =\left(\sum_{m \in Z} \eta_{m}\left(\prod_{j=2}^{n} q_{1 j}^{m_{j}}-\gamma_{1} q_{1 t}\right) x_{1}^{m_{1}+1} \cdots x_{n}^{m_{n}}\right) \\
& +\theta_{1 t} x_{1} x_{t}+K x_{t} .
\end{aligned}
$$

$\operatorname{As} \operatorname{Mon}\left(\mathcal{O}_{q, \delta}^{r, n}\right)$ is a basis of $\mathcal{O}_{q, \delta}^{r, n}$ and $\prod_{j=2}^{n} q_{j 1}^{m_{j}}-\gamma_{1} q_{1 t} \in A^{*}$ we have that $\eta_{m}=0$ if $m \neq(0, \ldots, 1, \ldots, 0)$ where 1 is in the $t$ th position, so $\bar{u}_{t}=\eta_{t} x_{t}$ and $K=0$. In the other hand

$$
0=\eta_{t} x_{t} x_{1}-q_{1 t} \gamma_{1} x_{1} \eta_{t} x_{t}+\theta_{1 t} x_{1} x_{t}=\left(\left(q_{1 t}-q_{1 t} \gamma_{1}\right) \eta_{t}+\theta_{1 t}\right) x_{1} x_{t}
$$

then $\left(q_{1 t}-q_{1 t} \gamma_{1}\right) \eta_{t}+\theta_{1 t}=0$.

2) Put $u_{1}=u_{1}^{\prime}+v_{1}$ with $v_{1} \in \mathcal{O}_{q, \delta}^{r, n} \backslash A\left(\left(x_{1}\right)\right)$. By the corollary $(2.5)$ there exists $w \in \mathcal{O}_{q, \delta}^{r, n}$ with $v_{1}=[\operatorname{ad} w] x_{1}$, we will denote $\bar{u}_{i}=u_{i}-[\operatorname{ad} w] x_{i}$ so we have $\bar{u}_{1} \in A\left(\left(x_{1}\right)\right)$. Let $t \neq 1$

$$
\bar{u}_{t} x_{1}-q_{1 t} x_{1} \bar{u}_{t}=-x_{t} \bar{u}_{1}+q_{1 t} \bar{u}_{1} x_{t}-\theta_{1 t} x_{1} x_{t}-K x_{t} \in A\left(\left(x_{1}\right)\right) x_{t}
$$

because if $\bar{u}_{1}=\sum_{t} \rho_{t} x_{1}^{t}$ then $x_{t} \bar{u}_{1}=\sum_{t} \rho_{t} q_{1 t}^{t} x_{1}^{t} x_{t}$. If

$$
\bar{u}_{t}=\sum_{m \in Z} \eta_{m} x_{1}^{m_{1}} \cdots x_{n}^{m_{n}}
$$

then

$$
\begin{aligned}
\bar{u}_{t} x_{1}-x_{1} q_{1 t} \bar{u}_{t} & =\sum_{m \in Z} \eta_{m} x_{1}^{m_{1}} \cdots x_{n}^{m_{n}} x_{1}-x_{1} q_{1 t} \sum_{m \in Z} \eta_{m} x_{1}^{m_{1}} \cdots x_{n}^{m_{n}} \\
& =\sum_{m \in Z} \eta_{m}\left(\prod_{j=2}^{n} q_{1 j}^{m_{j}}-q_{1 t}\right) x_{1} x_{1}^{m_{1}} \cdots x_{n}^{m_{n}} \\
& =x_{1} \sum_{m \in Z} \eta_{m}\left(\prod_{j=2}^{n} q_{1 j}^{m_{j}}-q_{1 t}\right) x_{1}^{m_{1}} \cdots x_{n}^{m_{n}}
\end{aligned}
$$

multiplying by $x_{1}^{-1}$ we have

$$
\sum_{m \in Z} \eta_{m}\left(\prod_{j=2}^{n} q_{1 j}^{m_{j}}-q_{1 t}\right) x_{1}^{m_{1}} \cdots x_{n}^{m_{n}} \in A\left(\left(x_{1}\right)\right) x_{t} .
$$

Since $\prod_{j=2}^{n} q_{1 j}^{m_{j}}-q_{1 t} \in A^{*}$ we have $\eta_{m}=0$ if $m_{i} \neq 0$ for $i \neq 1$, or if $m_{t} \neq 1$. Then $\bar{u}_{t}=f_{t}\left(x_{1}\right) x_{t}$ where $f_{t}\left(x_{1}\right) \in A\left(\left(x_{1}\right)\right)$. Take $t=2$, if 
$f_{2}\left(x_{1}\right)=\lambda_{2}+\sum_{t \neq 0} \rho_{t} x_{1}^{t}$ then for all $t$ put $w_{t}=\left(1-q_{12}^{t}\right)^{-1}$, note that $w_{t} x_{i}=x_{i} w_{t}$ for all $i=1, \ldots, n$ so

$$
\begin{aligned}
{\left[\operatorname{ad} w_{t} \rho_{t} x_{1}^{t}\right] x_{2} } & =w_{t} \rho_{t} x_{1}^{t} x_{2}-x_{2} w_{t} \rho_{t} x_{1}^{t} \\
& =w_{t}\left(\rho_{t} x_{1}^{t} x_{2}-x_{2} \rho_{t} x_{1}^{t}\right)=w_{t}\left(\rho_{t} x_{1}^{t} x_{2}-\rho_{t} x_{2} x_{1}^{t}-\delta_{2}\left(\rho_{t}\right) x_{1}^{t}\right) \\
& =w_{t}\left(1-q_{12}^{t}\right) \rho_{t} x_{1}^{t} x_{2}-w_{t} \delta_{2}\left(\rho_{t}\right) x_{1}^{t}=\rho_{t} x_{1}^{t} x_{2}-w_{t} \delta_{2}\left(\rho_{t}\right) x_{1}^{t} .
\end{aligned}
$$

Then there exists $w^{\prime} \in A\left(\left(x_{1}\right)\right) \backslash A$ or $w^{\prime}=0$ with $\bar{u}_{2}-\left[\operatorname{ad} w^{\prime}\right] x_{2}=$ $\lambda_{2} x_{2}+f^{\prime}\left(x_{1}\right)$ and $f^{\prime}\left(x_{1}\right) \in A\left(\left(x_{1}\right)\right) \backslash A$ or $f^{\prime}\left(x_{1}\right)=0$.

Take $\tilde{u}_{l}=\bar{u}_{l}-\left[\operatorname{ad} w^{\prime}\right] x_{l}=g_{l}\left(x_{1}\right) x_{l}+g_{l}^{\prime}\left(x_{1}\right),\left(g_{l}^{\prime}\left(x_{1}\right) \in A\left(\left(x_{1}\right)\right) \backslash A\right.$ or $\left.g_{l}^{\prime}\left(x_{1}\right)=0\right)$, because if $w^{\prime}=\sum_{t} a_{t} x_{1}^{t}$ then

$$
\begin{aligned}
{\left[\operatorname{ad} w^{\prime}\right] x_{l} } & =\sum_{t} a_{t} x_{1}^{t} x_{l}-\sum_{t} x_{l} a_{t} x_{1}^{t} \\
& =\sum_{t} a_{t} x_{1}^{t} x_{l}-\sum_{t} a_{t} q_{1 t}^{t} x_{1}^{t} x_{l}-\sum_{t} \delta_{l}\left(a_{t}\right) x_{1}^{t}
\end{aligned}
$$

and $\tilde{u}_{2}=\lambda_{2} x_{2}+g_{2}^{\prime}\left(x_{1}\right)$.

By the equation (1) for $(t=1)$ if $\tilde{u}_{1}=\sum_{t} a_{t} x_{1}^{t}$ then

$$
\begin{aligned}
0= & \lambda_{2} x_{2} x_{1}+g_{2}^{\prime}\left(x_{1}\right) x_{1}+x_{2} \tilde{u}_{1}-q_{12} \tilde{u}_{1} x_{2}-q_{12} x_{1} \lambda_{2} x_{2}-q_{12} x_{1} g_{2}^{\prime}\left(x_{1}\right) \\
& \quad+\theta_{12} x_{1} x_{2}+K x_{1} \\
= & \left.\lambda_{2} q_{12} x_{1} x_{2}+\sum_{t} x_{2} a_{t} x_{1}^{t}-\sum_{t} q_{12} a_{t} x_{1}^{t} x_{2}-q_{12} \lambda_{2} x_{1} x_{2}+\theta_{12} x_{1} x_{2}\right] \\
& \quad+\left[g_{2}^{\prime}\left(x_{1}\right) x_{1}+q_{12} x_{1} g_{2}^{\prime}\left(x_{1}\right)+K x_{1}\right] \\
= & \left(\lambda_{2} q_{12} x_{1} x_{2}+\sum_{t} a_{t} x_{2} x_{1}^{t}-\sum_{t} q_{12} a_{t} x_{1}^{t} x_{2}-q_{12} \lambda_{2} x_{1} x_{2}+\theta_{12} x_{1} x_{2}\right) \\
& \quad+\left(g_{2}^{\prime}\left(x_{1}\right) x_{1}-q_{12} x_{1} g_{2}^{\prime}\left(x_{1}\right)+K x_{1}+\sum_{t} \delta_{2}\left(a_{t}\right) x_{1}^{t}\right) \\
= & \left(\sum_{t} a_{t}\left(q_{12}^{t}-q_{12}\right) x_{1}^{t} x_{2}+\theta_{12} x_{1} x_{2}\right) \\
& +\left(g_{2}^{\prime}\left(x_{1}\right) x_{1}-q_{12} x_{1} g_{2}^{\prime}\left(x_{1}\right)+K x_{1}+\sum_{t} \delta_{2}\left(a_{t}\right) x_{1}^{t}\right) .
\end{aligned}
$$

Then $t=1, \theta_{12}=0$, and $\tilde{u}_{1}=a x_{1}$. For this equations if $g_{2}^{\prime}\left(x_{1}\right) \neq 0$ put it $\left(g_{2}^{\prime}\left(x_{1}\right)\right)=b_{v} x_{1}^{v}$ with $v \neq 0$ then

$$
0=b_{v} x_{1}^{v+1}-q_{12} b_{v} x_{1}^{v+1}=b_{v}\left(1-q_{12}\right) x_{1}^{v+1}
$$


so $b_{v}=0$ but this is not possible. Thus $g_{2}^{\prime}\left(x_{1}\right)=0$.

Take $3 \leqslant t$ and $\tilde{u}_{1}=a_{1} x_{1}$.

$$
\begin{aligned}
& 0=\left(g_{t}\left(x_{1}\right) x_{t}+g_{t}^{\prime}\left(x_{1}\right)\right) x_{1}+x_{t} a_{1} x_{1}-q_{1 t} a_{1} x_{1} x_{t} \\
& \quad-q_{1 t} x_{1}\left(g_{t}\left(x_{1}\right) x_{t}+g_{t}^{\prime}\left(x_{1}\right)\right)+\theta_{1 t} x_{1} x_{t}+K x_{1} \\
&=\left(g_{t}\left(x_{1}\right) x_{t} x_{1}+a_{1} x_{t} x_{1}-q_{1 t} a_{1} x_{1} x_{t}-q_{1 t} x_{1} g_{t}\left(x_{1}\right) x_{t}+\theta_{1 t} x_{1} x_{t}\right) \\
& \quad \quad\left(g_{t}^{\prime}\left(x_{1}\right) x_{1}+\delta_{t}\left(a_{1}\right) x_{1}-q_{1 t} x_{1} g_{t}^{\prime}\left(x_{1}\right)+K x_{1}\right) \\
&=\left(g_{t}\left(x_{1}\right) x_{t} x_{1}-q_{1 t} x_{1} g_{t}\left(x_{1}\right) x_{t}+\theta_{1 t} x_{1} x_{t}\right) \\
& \quad \quad\left(g_{t}^{\prime}\left(x_{1}\right) x_{1}+\delta_{t}\left(a_{1}\right) x_{1}-q_{1 t} x_{1} g_{t}^{\prime}\left(x_{1}\right)+K x_{1}\right) \\
&=\theta_{1 t} x_{1} x_{t}+\left(g_{t}^{\prime}\left(x_{1}\right) x_{1}+\delta_{t}\left(a_{1}\right) x_{1}-q_{1 t} x_{1} g_{t}^{\prime}\left(x_{1}\right)+K x_{1}\right) .
\end{aligned}
$$

Then $\theta_{1 t}=0$ and if $g_{t}^{\prime}\left(x_{1}\right) \neq 0$ we can take $i t\left(g_{t}^{\prime}\left(x_{1}\right)\right)=b_{v} x_{1}^{v}$ with $b_{v} \neq 0$. Of this equation we can claim that $b_{v}\left(1-q_{1 t}\right)=0$ then $b_{v}=0$ but this is not possible so $g_{t}^{\prime}\left(x_{1}\right)=0$.

In the other hand, if $g_{t}\left(x_{1}\right)=\sum_{t} c_{t} x_{1}^{t}$

$$
\begin{aligned}
& 0=g_{t}\left(x_{1}\right) x_{t} x_{2}+x_{t} \lambda_{2} x_{2}-q_{2 t} \lambda_{2} x_{2} x_{t}-q_{2 t} x_{2} g_{t}\left(x_{1}\right) x_{t}+\theta_{2 t} x_{2} x_{t} \\
& +K x_{2}+K^{\prime} x_{t}+\hat{\theta}_{2 t} p_{2 t}\left(x_{1}, \ldots, x_{n}\right)-\bar{p}_{2 t}\left(\tilde{u}_{1}, \ldots, \tilde{u}_{n}\right)+a_{2 t}^{(0)} \theta \\
& =g_{t}\left(x_{1}\right) x_{t} x_{2}+\left(\lambda_{2} x_{t} x_{2}-\lambda_{2} q_{2 t} x_{2} x_{t}\right)-q_{2 t} x_{2} g_{t}\left(x_{1}\right) x_{t}+\theta_{2 t} x_{2} x_{t}+K x_{2} \\
& +K^{\prime} x_{t}+\hat{\theta}_{2 t} p_{2 t}\left(x_{1}, \ldots, x_{n}\right)-\bar{p}_{2 t}\left(\tilde{u}_{1}, \ldots, \tilde{u}_{n}\right)+\delta_{t}\left(\lambda_{2}\right) x_{2}+a_{2 t}^{(0)} \theta \\
& =g_{t}\left(x_{1}\right)\left(q_{2 t} x_{2} x_{t}+p_{2 t}\left(x_{1}, \ldots, x_{n}\right)\right)-q_{2 t} x_{2} \sum_{t} c_{t} x_{1}^{t} x_{t}+\theta_{2 t} x_{2} x_{t} \\
& +\lambda_{2} p_{2 t}\left(x_{1}, \ldots, x_{n}\right)+K x_{2}+K^{\prime} x_{t}+\hat{\theta}_{2 t} p_{2 t}\left(x_{1}, \ldots, x_{n}\right) \\
& -\bar{p}_{2 t}\left(\tilde{u}_{1}, \ldots, \tilde{u}_{n}\right)+\delta_{t}\left(\lambda_{2}\right) x_{2}+a_{2 t}^{(0)} \theta \\
& =q_{2 t}\left(\sum_{t} c_{t} x_{1}^{t} x_{2} x_{t}-\sum_{t} c_{t} x_{2} x_{1}^{t} x_{t}\right)+\theta_{2 t} x_{2} x_{t}+g_{t}\left(x_{1}\right) p_{2 t}\left(x_{1}, \ldots, x_{n}\right) \\
& +\sum_{t} \delta_{2}\left(c_{t}\right) x_{1}^{t} x_{t}+K x_{2}+K^{\prime} x_{t}+\left(\hat{\theta}_{2 t}+\lambda_{2}\right) p_{2 t}\left(x_{1}, \ldots, x_{n}\right) \\
& -\bar{p}_{2 t}\left(\tilde{u}_{1}, \ldots, \tilde{u}_{n}\right)+\delta_{t}\left(\lambda_{2}\right) x_{2}+a_{2 t}^{(0)} \theta \\
& =q_{2 t}\left(\sum_{t} c_{t}\left(1-q_{12}^{t}\right) x_{1}^{t} x_{2} x_{t}\right)+\theta_{2 t} x_{2} x_{t}+g_{t}\left(x_{1}\right) p_{2 t}\left(x_{1}, \ldots, x_{n}\right) \\
& +\sum_{t} \delta_{2}\left(c_{t}\right) x_{1}^{t} x_{t}+K x_{2}+K^{\prime} x_{t}+\left(\hat{\theta}_{2 t}+\lambda_{2}\right) p_{2 t}\left(x_{1}, \ldots, x_{n}\right) \\
& -\bar{p}_{2 t}\left(\tilde{u}_{1}, \ldots, \tilde{u}_{n}\right)+\delta_{t}\left(\lambda_{2}\right) x_{2}+a_{2 t}^{(0)} \theta \text {. }
\end{aligned}
$$


Of this last equation we have that $c_{t}\left(1-q_{12}^{t}\right)=0$ for $t \neq 0$ so $c_{t}=0$ since $\left(1-q_{12}^{t}\right) \in A^{*}$, thus $g_{t}\left(x_{1}\right)=\lambda_{t}$ of this way $u_{t}-\left[\operatorname{ad} w+w^{\prime}\right] x_{t}=\tilde{u}_{t}=$ $\lambda_{t} x_{t}$.

\section{Poisson brackets on $\mathcal{O}_{q, \delta}^{r, n}$}

Definition 3.1. A Poisson bracket $\{\cdot, \cdot\}$ is a $\mathbb{Z}$-bilinear function on $\mathcal{O}_{q, \delta}^{r, n}$ such that

1) $\{\cdot, \cdot\}$ is a Lie bracket.

2) $\{a b, c\}=\{a, c\} b+a\{b, c\}$ for all $a, b, c \in \mathcal{O}_{q, \delta}^{r, n}$.

3) $0=\{a, b\}+\{b, a\}$ for all $a, b \in \mathcal{O}_{q, \delta}^{r, n}$.

Remark 3.2. If $\partial$ is a derivation on $\mathcal{O}_{q, \delta}^{r, n}$ then for all $i=1, \ldots, r$ we have $0=\partial(1)=\partial\left(x_{i} x_{i}^{-1}\right)=\partial\left(x_{i}\right) x_{i}^{-1}+x_{i} \partial\left(x_{i}^{-1}\right)$ then $\partial\left(x_{i}^{-1}\right)=$ $-x_{i}^{-1} \partial\left(x_{i}\right) x_{i}^{-1}$.

Proposition 3.3. Let be given a Poisson bracket $\{a, b\}$ on an extension $\mathcal{O}_{q, \delta}^{r, n}$ of $\mathrm{A}$. Then there exists $\xi, \xi_{j} \in A$ such that $\left\{x_{i}, x_{j}\right\}=\xi\left[x_{i}, x_{j}\right]+$ $\delta_{i}(\xi) x_{i}-\delta_{i}(\xi) x_{j}-\delta_{i}\left(\xi_{j}\right)$ with $\delta_{j}\left(\xi_{j}\right)=0$ for all $j, i=1, \ldots, n$, where $[a, b]:=a b-b a$.

Proof. By theorem (2.6) for all $a \in \mathcal{O}_{q, \delta}^{r, n}$ there exists $\lambda_{a}\left(x_{i}\right) \in A$ and $w(a) \in \mathcal{O}_{q, \delta}^{r, n}$ with

$$
\left\{x_{i}, a\right\}=\lambda_{a}\left(x_{i}\right) x_{i}+[\operatorname{ad} w(a)] x_{i} .
$$

So we have

$$
0=\left\{x_{i}, x_{i}\right\}=\lambda_{i}\left(x_{i}\right) x_{i}+\left[\operatorname{ad} w\left(x_{i}\right)\right] x_{i} .
$$

Put $w\left(x_{i}\right)=\sum_{m=0}^{k} \eta_{v_{m}} x_{1}^{v_{m_{1}}} \cdots x_{n}^{v_{m_{n}}}$, we will see that $w\left(x_{i}\right) \in A\left(\left(x_{i}\right)\right)$ for $i=1, \ldots, r$ or $w\left(x_{i}\right) \in A\left[x_{i}\right]$ for $i=r+1, \ldots, n$. If $w\left(x_{i}\right)=0$ we have the claimed, in the other hand,

$$
\begin{aligned}
0= & \lambda_{i}\left(x_{i}\right) x_{i}-\sum_{m=0}^{k} x_{i} \eta_{v_{m}} x_{1}^{v_{m_{1}}} \cdots x_{n}^{v_{m_{n}}}+\sum_{m=0}^{k} \eta_{v_{m}} x_{1}^{v_{m_{1}}} \cdots x_{n}^{v_{m_{n}}} x_{i} \\
= & \lambda_{i}\left(x_{i}\right) x_{i}-\sum_{m=0}^{k} \delta_{i}\left(\eta_{v_{m}}\right) x_{1}^{v_{m_{1}}} \cdots x_{n}^{v_{m_{n}}}-\sum_{m=0}^{k} \eta_{v_{m}} x_{i} x_{1}^{v_{m_{1}}} \cdots x_{n}^{v_{m_{n}}} \\
& +\sum_{m=0}^{k} \eta_{v_{m}} x_{1}^{v_{m_{1}}} \cdots x_{n}^{v_{m_{n}}} x_{i}
\end{aligned}
$$




$$
\begin{aligned}
& =\lambda_{i}\left(x_{i}\right) x_{i}-\sum_{m=0}^{k} \delta_{i}\left(\eta_{v_{m}}\right) x_{1}^{v_{m_{1}}} \cdots x_{n}^{v_{m_{n}}} \\
& \quad-\sum_{m=0}^{k}\left(\eta_{v_{m}} \prod_{s<i} q_{s i}^{v_{m_{s}}} x_{1}^{v_{m_{1}}} \cdots x_{i}^{v_{m_{i}}+1} \cdots x_{n}^{v_{m_{n}}}+p_{v_{m}, i}\left(x_{1}, \ldots, x_{n}\right)\right) \\
& \quad+\sum_{m=0}^{k}\left(\eta_{v_{m}} \prod_{s>i} q_{i s}^{v_{m_{s}}} x_{1}^{v_{m_{1}}} \cdots x_{i}^{v_{m_{i}}+1} \cdots x_{n}^{v_{m_{n}}}+p_{i, v_{m}}\left(x_{1}, \ldots, x_{n}\right)\right) \\
& =\sum_{m=0}^{k} \eta_{v_{m}}\left(\prod_{s>i} q_{i s}^{v_{m_{s}}}-\prod_{s<i} q_{s i}^{v_{m_{s}}}\right) x_{1}^{v_{m_{1}}} \cdots x_{i}^{v_{m_{i}}+1} \cdots x_{n}^{v_{m_{n}}} \\
& \quad+\lambda_{i}\left(x_{i}\right) x_{i}-\sum_{m=0}^{k} \delta_{i}\left(\eta_{v_{m}}\right) x_{1}^{v_{m_{1}}} \cdots x_{n}^{v_{m_{n}}}+\sum_{m=0}^{k} p_{i, v_{m}}\left(x_{1}, \ldots, x_{n}\right) \\
& \quad-p_{v_{m}, i}\left(x_{1}, \ldots, x_{n}\right),
\end{aligned}
$$

where $\operatorname{deg}\left(p_{v_{m}, i}\right), \operatorname{deg}\left(p_{i, v_{m}}\right)<v_{m_{1}}+\cdots+v_{m_{n}}+1<\left|v_{k}\right|+1$ for all $m=0, \ldots, k$. By the last equation we have $\left(\prod_{s<i} q_{s i}^{v_{k_{s}}}-\prod_{s>i} q_{i s}^{v_{k_{s}}}\right) \eta_{v_{k}}=0$ and since $\eta_{v_{k}} \neq 0$ then $v_{k_{l}}=0$ for $l \neq i$, because if there exists $v_{k_{l}} \neq 0$ for some $l \neq i$ then $\prod_{s<i} q_{s i}^{v_{m_{s}}}-\prod_{s>i} q_{i s}^{v_{m_{s}}} \in A^{*}$ by definition of $\mathcal{O}_{q, \delta}^{r, n}$, so this implies that $\eta_{v_{k}}=0$, but this is not possible. Later $p_{i, v_{k}}=p_{v_{k}, i}=0$ and

$$
\begin{aligned}
0= & \sum_{m=0}^{k-1} \eta_{v_{m}}\left(\prod_{s>i} q_{i s}^{v_{m_{s}}}-\prod_{s<i} q_{s i}^{v_{m_{s}}}\right) x_{1}^{v_{m_{1}}} \cdots x_{i}^{v_{m_{i}}+1} \cdots x_{n}^{v_{m_{n}}}+\lambda_{i}\left(x_{i}\right) x_{i} \\
& \quad-\sum_{m=0}^{k-1} \delta_{i}\left(\eta_{v_{m}}\right) x_{1}^{v_{m_{1}}} \cdots x_{n}^{v_{m_{n}}}+\sum_{m=0}^{k-1} p_{i, v_{m}}\left(x_{1}, \ldots, x_{n}\right) \\
& \quad-p_{v_{m}, i}\left(x_{1}, \ldots, x_{n}\right) .
\end{aligned}
$$

So $\eta_{v_{k-1}}=0$, if $\eta_{v_{k-1}} \neq 0$ then $v_{k-1_{l}}=0$ for $l \neq i$ because $\left(\prod_{s<i} q_{s i}^{v_{k-1}}-\right.$ $\left.\prod_{s>i} q_{i s}^{v_{k-1}}\right) \in A^{*}$ if there exist $v_{k-1_{l}} \neq 0$ for some $l \neq i$. By recurrently way we can claim that $\eta_{v_{m}}=0$ if there exists $v_{m_{l}} \neq 0$ for some $l \neq i$, then we have the claimed. Since $w\left(x_{i}\right)=\sum_{t \in \mathbb{Z}} \xi_{i t} x_{i}^{t}$, where $\xi_{i t}=0$ if $t<0$ and $i>r$, then

$$
0=\lambda_{i}\left(x_{i}\right) x_{i}-x_{i} \sum_{t \in Z} \xi_{i t} x_{i}^{t}+\sum_{t \in \mathbb{Z}} \xi_{i t} x_{i}^{t+1}=\lambda_{i}\left(x_{i}\right) x_{i}-\sum_{t \in \mathbb{Z}} \delta_{i}\left(\xi_{i t}\right) x_{i}^{t}
$$


then $\delta_{i}\left(\xi_{i t}\right)=0$ for $t \neq 1$ and $\lambda_{i}\left(x_{i}\right)=\delta_{i}\left(\xi_{i 1}\right)$. Put $i<j, w\left(x_{i}\right)=$ $\sum_{l=0}^{k} \xi_{i v_{i}} x_{i}^{v_{i_{l}}}$, and $w\left(x_{j}\right)=\sum_{l=0}^{r} \xi_{j v_{j_{l}}} x_{j}^{v_{j_{l}}}$, so

$$
\begin{aligned}
& 0=\left\{x_{j}, x_{i}\right\}+\left\{x_{i}, x_{j}\right\}=\lambda_{i}\left(x_{j}\right) x_{j}+\left[\operatorname{ad} w\left(x_{i}\right)\right] x_{j}+\lambda_{j}\left(x_{i}\right) x_{i}+\left[\operatorname{ad} w\left(x_{j}\right)\right] x_{i} \\
& =\lambda_{i}\left(x_{j}\right) x_{j}+\lambda_{j}\left(x_{i}\right) x_{i}+\sum_{l=0}^{k} \xi_{i v_{i_{l}}} x_{i}^{v_{i_{l}}} x_{j}-x_{j} \sum_{l=0}^{k} \xi_{i v_{i_{l}}} x_{i}^{v_{i_{l}}}+\sum_{l=0}^{r} \xi_{j v_{j_{l}}} x_{j}^{v_{j_{l}}} x_{i} \\
& -x_{i} \sum_{l=0}^{r} \xi_{j v_{j_{l}}} x_{j}^{v_{j_{l}}} \\
& =\lambda_{i}\left(x_{j}\right) x_{j}+\lambda_{j}\left(x_{i}\right) x_{i}+\xi_{i v_{i_{k}}} x_{i}^{v_{i_{k}}} x_{j}-\xi_{i v_{i_{k}}} x_{j} x_{i}^{v_{i_{k}}}+\xi_{j v_{j_{r}}} x_{j}^{v_{j_{r}}} x_{i} \\
& -\xi_{j v_{j_{r}}} x_{i} x_{j}^{v_{j_{r}}}+\sum_{l=0}^{k-1} \xi_{i v_{i}} x_{i}^{v_{i_{l}}} x_{j}-\sum_{l=0}^{k-1} \xi_{i v_{i_{l}}} x_{j} x_{i}^{v_{i_{l}}}+\sum_{l=0}^{r-1} \xi_{j v_{j_{l}}} x_{j}^{v_{j_{l}}} x_{i} \\
& -\sum_{l=0}^{r-1} \xi_{j v_{j_{l}}} x_{i} x_{j}^{v_{j_{l}}}-\sum_{l=0}^{k-1} \delta_{j}\left(\xi_{i v_{i_{l}}}\right) x_{i}^{v_{i_{l}}}-\sum_{l=0}^{r-1} \delta_{i}\left(\xi_{j v_{j_{l}}}\right) x_{j}^{v_{j_{l}}}-\delta_{j}\left(\xi_{i v_{i_{k}}}\right) x_{i}^{v_{i_{k}}} \\
& -\delta_{i}\left(\xi_{j v_{j_{r}}}\right) x_{j}^{v_{j_{r}}} \\
& =\lambda_{i}\left(x_{j}\right) x_{j}+\lambda_{j}\left(x_{i}\right) x_{i}+\xi_{i v_{i}} x_{i}^{v_{i_{k}}} x_{j}-\xi_{i v_{i_{k}}} q_{i j}^{v_{i_{k}}} x_{i}^{v_{i_{k}}} x_{j}+\xi_{j v_{j_{r}}} q_{i j}^{v_{j_{r}}} x_{i} x_{j}^{v_{j_{r}}} \\
& -\xi_{j v_{j_{r}}} x_{i} x_{j}^{v_{j_{r}}}+\sum_{l=0}^{k-1} \xi_{i v_{i}} x_{i}^{v_{i_{l}}} x_{j}-\sum_{l=0}^{k-1} \xi_{i v_{i_{l}}} q_{i j}^{v_{i_{l}}} x_{i}^{v_{i_{l}}} x_{j}+\sum_{l=0}^{r-1} \xi_{j v_{j_{l}}} q_{i j}^{v_{j_{l}}} x_{i} x_{j}^{v_{j_{l}}} \\
& -\sum_{l=0}^{r-1} \xi_{j v_{j_{l}}} x_{i} x_{j}^{v_{j_{l}}}-\sum_{l=0}^{k} \delta_{j}\left(\xi_{i v_{i_{l}}}\right) x_{i}^{v_{i_{l}}}-\sum_{l=0}^{r} \delta_{i}\left(\xi_{j v_{j_{l}}}\right) x_{j}^{v_{j_{l}}}-\xi_{i v_{i_{k}}} p_{v_{i_{k}}, j} \\
& +\xi_{j v_{j r}} p_{i, v_{j r}}-\sum_{l=0}^{k-1} \xi_{i v_{i_{l}}} p_{v_{i_{l}}, j}+\sum_{l=0}^{r-1} \xi_{j v_{j_{l}}} p_{i, v_{j_{l}}} \\
& =\lambda_{i}\left(x_{j}\right) x_{j}+\lambda_{j}\left(x_{i}\right) x_{i}+\xi_{i v_{i_{k}}}\left(1-q_{i j}^{v_{i k}}\right) x_{i}^{v_{i k}} x_{j}+\xi_{j v_{j_{r}}}\left(q_{i j}^{v_{j_{r}}}-1\right) x_{i} x_{j}^{v_{j_{r}}} \\
& +\sum_{l=0}^{k-1} \xi_{i v_{i_{l}}}\left(1-q_{i j}^{v_{i_{l}}}\right) x_{i}^{v_{i_{l}}} x_{j}+\sum_{l=0}^{r-1} \xi_{j v_{j_{l}}}\left(q_{i j}^{v_{j_{l}}}-1\right) x_{i} x_{j}^{v_{j_{l}}} \\
& -\sum_{l=0}^{k} \delta_{j}\left(\xi_{i v_{i_{l}}}\right) x_{i}^{v_{i_{l}}}-\sum_{l=0}^{r} \delta_{i}\left(\xi_{j v_{j_{l}}}\right) x_{j}^{v_{j_{l}}}-\sum_{l=0}^{k} \xi_{i v_{i}} p_{v_{i_{l}}, j}+\sum_{l=0}^{r} \xi_{j v_{j_{l}}} p_{i, v_{j_{l}}}
\end{aligned}
$$

where $\operatorname{deg}\left(p_{v_{i_{l}}, j}\right)<v_{i_{l}}+1<v_{i_{k}}+1$ and $\operatorname{deg}\left(p_{i, v_{j_{l}}}\right)<v_{j_{l}}+1<v_{j_{r}}+1$ for all $l$. Since $0=\xi_{i v_{i_{k}}}\left(1-q_{i j}^{v_{i_{k}}}\right) x_{i}^{v_{i_{k}}} x_{j}+\xi_{j v_{j_{r}}}\left(q_{i j}^{v_{j_{r}}}-1\right) x_{i} x_{j}^{v_{j_{r}}}$, we have $v_{j_{r}}=v_{i_{k}}$ and $v_{j_{r}} \in\{0,1\}$, later $p_{v_{i_{k}}, j}, p_{i, v_{j_{r}}} \in A+A x_{1}+\cdots+A x_{n}$. The 
same way

$$
\begin{aligned}
0=\lambda_{i}\left(x_{j}\right) x_{j}+\lambda_{j}\left(x_{i}\right) x_{i}+\xi_{i v_{i_{k-1}}}\left(1-q_{i j}^{v_{i_{k-1}}}\right) x_{i}^{v_{i_{k-1}}} x_{j} \\
\quad+\xi_{j v_{j_{r-1}}}\left(q_{i j}^{v_{j_{r-1}}}-1\right) x_{i} x_{j}^{v_{j_{r}-1}}+\sum_{l=0}^{k-2} \xi_{i v_{i_{l}}}\left(1-q_{i j}^{v_{i_{l}}}\right) x_{i}^{v_{i_{l}}} x_{j} \\
\quad+\sum_{l=0}^{r-2} \xi_{j v_{j_{l}}}\left(q_{i j}^{v_{j_{l}}}-1\right) x_{i} x_{j}^{v_{j_{l}}}-\sum_{l=0}^{k} \delta_{j}\left(\xi_{i v_{i_{l}}}\right) x_{i}^{v_{i_{l}}}-\sum_{l=0}^{r} \delta_{i}\left(\xi_{j v_{j_{l}}}\right) x_{j}^{v_{j_{l}}} \\
\quad-\sum_{l=0}^{k} \xi_{i v_{i_{l}}} p_{v_{i_{l}}, j}+\sum_{l=0}^{r} \xi_{j v_{j_{l}}} p_{i, v_{j_{l}}} .
\end{aligned}
$$

Then $0=\xi_{i v_{i_{k-1}}}\left(1-q_{i j}^{v_{i_{k-1}}}\right) x_{i}^{v_{i_{k-1}}} x_{j}+\xi_{j v_{j_{r-1}}}\left(q_{i j}^{v_{j_{r-1}}}-1\right) x_{i} x_{j}^{v_{j_{r-1}}}$, since $v_{i_{k-1}}, v_{j_{r-1}} \leqslant 0, \xi_{j v_{j_{k-1}}}, \xi_{i v_{i_{r-1}}} \neq 0$, and $\left(1-q_{i j}^{v_{i_{k}}}\right),\left(q_{i j}^{v_{j_{k}}}-1\right) \in A^{*}$ if $v_{i_{k-1}}, v_{j_{r-1}} \neq 0$ then we can claim that $v_{i_{k-1}}, v_{j_{r-1}}=0$. Of recurrently form we can claim that $w\left(x_{t}\right)=\xi_{t 1} x_{t}+\xi_{t 0}$ with $\xi_{t 1}, \xi_{t 0} \in A$ and $\delta_{t}\left(\xi_{t 0}\right)=0$ for all $t=1, \ldots, n$. Again put $i<j$

$$
\begin{aligned}
0=\left\{x_{j}, x_{i}\right\}+\left\{x_{i}, x_{j}\right\}=\lambda_{i}\left(x_{j}\right) x_{j}+\left[\operatorname{ad} w\left(x_{i}\right)\right] x_{j}+\lambda_{j}\left(x_{i}\right) x_{i}+\left[\operatorname{ad} w\left(x_{j}\right)\right] x_{i} \\
=\lambda_{i}\left(x_{j}\right) x_{j}+\lambda_{j}\left(x_{i}\right) x_{i}+\xi_{i 1} x_{i} x_{j}+\xi_{i 0} x_{j}-x_{j} \xi_{i 1} x_{i}-x_{j} \xi_{i 0} \\
\quad+\xi_{j 1} x_{j} x_{i}+\xi_{j 0} x_{i}-x_{i} \xi_{j 1} x_{j}-x_{i} \xi_{j 0} \\
=\lambda_{i}\left(x_{j}\right) x_{j}+\lambda_{j}\left(x_{i}\right) x_{i}+\xi_{i 1} x_{i} x_{j}+\xi_{i 0} x_{j} \\
\quad-\left(\delta_{j}\left(\xi_{i 1}\right) x_{i}+\xi_{i 1} q_{i j} x_{i} x_{j}+\xi_{i 1} p_{i j}\left(x_{1}, \ldots, x_{n}\right)\right) \\
\quad-\left(\xi_{i 0} x_{j}+\delta_{j}\left(\xi_{i 0}\right)\right)+\left(\xi_{j 1} q_{i j} x_{i} x_{j}+\xi_{j 1} p_{i j}\left(x_{1}, \ldots, x_{n}\right)\right) \\
\quad+\xi_{j 0} x_{i}-\left(\delta_{i}\left(\xi_{j 1}\right) x_{j}+\xi_{j 1} x_{i} x_{j}\right)-\left(\delta_{i}\left(\xi_{j 0}\right)+\xi_{j 0} x_{i}\right) \\
=\xi_{i 1} x_{i} x_{j}-\xi_{i 1} q_{i j} x_{i} x_{j}+\xi_{j 1} q_{i j} x_{i} x_{j}-\xi_{j 1} x_{i} x_{j} \\
\quad+\lambda_{i}\left(x_{j}\right) x_{j}+\lambda_{j}\left(x_{i}\right) x_{i}-\delta_{j}\left(\xi_{i 1}\right) x_{i}-\delta_{j}\left(\xi_{i 0}\right)-\xi_{i 1} p_{i j}\left(x_{1}, \ldots, x_{n}\right) \\
\quad+\xi_{j 1} p_{i j}\left(x_{1}, \ldots, x_{n}\right)-\delta_{i}\left(\xi_{j 1}\right) x_{j}-\delta_{i}\left(\xi_{j 0}\right) .
\end{aligned}
$$

So $\left(\xi_{i 1}-\xi_{j 1}\right)\left(1-q_{i j}\right)=0$ as $1-q_{i j} \in A^{*}$ then $\xi_{1 j}=\xi_{1 i}=\xi$. Later

$$
0=\left(\lambda_{i}\left(x_{j}\right)-\delta_{j}(\xi)\right) x_{j}+\left(\lambda_{j}\left(x_{i}\right)-\delta_{i}(\xi)\right)\left(x_{i}\right) x_{i}-\delta_{j}\left(\xi_{i 0}\right)-\delta_{i}\left(\xi_{j 0}\right)
$$

and $\lambda_{i}\left(x_{j}\right)=\delta_{j}(\xi), \lambda_{j}\left(x_{i}\right)=\delta_{i}(\xi), \delta_{j}\left(\xi_{i 0}\right)=-\delta_{i}\left(\xi_{j 0}\right)$. Note that

$$
\begin{aligned}
\left\{x_{i}, x_{j}\right\}= & \lambda_{j}\left(x_{i}\right) x_{i}+\left[\operatorname{ad} \xi x_{j}+\xi_{j 0}\right] x_{i}=\lambda_{j}\left(x_{i}\right) x_{i}+\xi x_{j} x_{i}+\xi_{j 0} x_{i} \\
& \quad-x_{i} \xi x_{j}-x_{i} \xi_{j 0} \\
= & \delta_{i}(\xi) x_{i}+\xi x_{j} x_{i}+\xi_{j 0} x_{i}-\xi x_{i} x_{j}+\delta_{i}(\xi) x_{j}-\xi_{j 0} x_{i}-\delta_{i}\left(\xi_{j 0}\right) \\
= & \xi\left[x_{i}, x_{j}\right]+\delta_{i}(\xi) x_{i}-\delta_{i}(\xi) x_{j}-\delta_{i}\left(\xi_{j 0}\right) .
\end{aligned}
$$


Lemma 3.4. Let $\{\cdot, \cdot\}$ be a Poisson bracket on $\mathcal{O}_{q, \delta}^{r, n}$ where $\delta_{i}=0$ for all $i=1, \ldots, n$. Then there exists $\xi \in A$ such that $\left\{x_{i}, a\right\}=\xi\left[x_{i}, a\right]$ for all $i=1, \ldots, n$ and $a=x_{t_{1}}^{b_{1}} \cdots x_{t_{n}}^{b_{n}} \in \mathcal{O}_{q, \delta}^{r, n}$ monomial.

Proof. Let $\xi \in A$ such that $\left\{x_{i}, x_{j}\right\}=\xi\left[x_{i}, x_{j}\right]$ for all $i, j=1, \ldots, n$ which is given by proposition (3.3) and $a=x_{t_{1}}^{b_{1}} \cdots x_{t_{l}}^{b_{l}}$ with $b_{r} \neq 0$ for all $r=1, \ldots, l$. Fix $i=1, \ldots, n$, we will show, by induction on $l$, that $\left\{a, x_{i}\right\}=\xi\left[a, x_{i}\right]$.

1) $\left(a=x_{t}^{n}\right)$ For this case we will do induction on $n$.

(a) $\left(a=x_{t}\right)$ we have $\left\{x_{t}, x_{i}\right\}=\xi\left[x_{t}, x_{i}\right]$ by the above proposition.

(b) $\left(a=x_{t}^{n+1}\right)$

$$
\begin{gathered}
\left\{x_{t}^{n+1}, x_{i}\right\}=\left\{x_{t}^{n}, x_{i}\right\} x_{t}+x_{t}^{n}\left\{x_{t}, x_{i}\right\}=\left(\xi\left[x_{t}^{n}, x_{i}\right]\right) x_{t}+x_{t}^{n}\left(\xi\left[x_{t}, x_{i}\right]\right) \\
=\xi\left(-x_{i} x_{t}^{n+1}+x_{t}^{n} x_{i} x_{t}-x_{t}^{n} x_{i} x_{t}+x_{t}^{n+1} x_{i}\right)=\xi\left[x_{t}^{n+1}, x_{i}\right]
\end{gathered}
$$

(c) $\left(a=x_{t}^{-1}\right.$, if $\left.t \leqslant r\right)$

$$
\begin{aligned}
& \left\{x_{t}^{-1}, x_{i}\right\}=-x_{t}^{-1}\left\{x_{t}, x_{i}\right\} x_{t}^{-1}=-\xi x_{t}^{-1}\left(\left[x_{t}, x_{i}\right]\right) x_{t}^{-1} \\
& \quad=\xi\left(-x_{t}^{-1}\left(x_{t} x_{i}-x_{i} x_{t}\right) x_{t}^{-1}\right)=\xi\left(x_{t}^{-1} x_{i}-x_{i} x_{t}^{-1}\right)=\xi\left[x_{t}^{-1}, x_{i}\right]
\end{aligned}
$$

(d) $\left(a=x_{t}^{-n-1}\right.$, if $\left.t \leqslant r\right)$

$$
\begin{aligned}
\left\{x_{t}^{-1-n}, x_{i}\right\} & =\left\{x_{t}^{-n}, x_{i}\right\} x_{t}^{-1}+x_{t}^{-n}\left\{x_{t}^{-1}, x_{i}\right\} \\
& =\xi\left(\left(\left[x_{t}^{-n}, x_{i}\right]\right) x_{t}^{-1}+x_{t}^{-n}\left(\left[x_{t}^{-1}, x_{i}\right]\right)\right) \\
& =\xi\left(-x_{i} x_{t}^{-n-1}+x_{t}^{-n} x_{i} x_{t}^{-1}-x_{t}^{-n} x_{i} x_{t}^{-1}+x_{t}^{-1-n} x_{i}\right) \\
& =\xi\left[x_{t}^{-n-1}, x_{i}\right] .
\end{aligned}
$$

2) $\left(a=x_{t_{1}}^{b_{1}} \cdots x_{t_{l}}^{b_{l}}\right)$

$$
\begin{aligned}
\left\{a, x_{i}\right\} & =\left\{x_{t_{1}}^{b_{1}} \cdots x_{t_{l}}^{b_{l}}, x_{i}\right\}=\left\{x_{t_{1}}^{b_{1}}, x_{i}\right\} x_{t_{2}}^{b_{2}} \cdots x_{t_{l}}^{b_{l}}+x_{t_{1}}^{b_{1}}\left\{x_{t_{2}}^{b_{2}} \cdots x_{t_{l}}^{b_{l}}, x_{i}\right\} \\
& =\xi\left(\left(\left[x_{t_{1}}^{b_{1}}, x_{i}\right]\right) x_{t_{2}}^{b_{2}} \cdots x_{t_{l}}^{b_{l}}+x_{t_{1}}^{b_{1}}\left(\left[x_{t_{2}}^{b_{2}} \cdots x_{t_{l}}^{b_{l}}, x_{i}\right]\right)\right) \\
& =\xi\left(-x_{i} a+x_{t_{1}}^{b_{1}} x_{i} x_{t_{2}}^{b_{2}} \cdots x_{t_{l}}^{b_{l}}-x_{t_{1}}^{b_{1}} x_{i} x_{t_{2}}^{b_{2}} \cdots x_{t_{l}}^{b_{l}}+a x_{i}\right)=\xi\left[a, x_{i}\right] .
\end{aligned}
$$

So we have the claimed. By theorem (2.6)

$$
\begin{aligned}
0 & =\left\{x_{i}, a\right\}+\left\{a, x_{i}\right\}=\lambda_{a}\left(x_{i}\right) x_{i}+[\operatorname{ad} w(a)] x_{i}+\left\{a, x_{i}\right\} \\
& =\lambda_{a}\left(x_{i}\right) x_{i}+[\operatorname{ad} w(a)] x_{i}-\xi[\operatorname{ad} a] x_{i}=\lambda_{a}\left(x_{i}\right) x_{i}+[\operatorname{ad} w(a)-\xi a] x_{i}
\end{aligned}
$$

but if we take $w(a)-\xi a$ as $w\left(x_{i}\right)$ in the proof of the above proposition, we can show that $w(a)-\xi a \in A\left(\left(x_{i}\right)\right)$ (if $\left.i=1, \ldots, r\right)$ or $w(a)-\xi a \in A\left[x_{i}\right]$ (if 
$i=r+1, \ldots, n)$ for all $i=1, \ldots, n$ then $w(a)-\xi a=l$ with $l \in A$. Then $[\operatorname{ad} w(a)] x_{i}=[\operatorname{ad} \xi a+l] x_{i}=[\operatorname{ad} \xi a] x_{i}+[\operatorname{ad} l] x_{i}=(\xi a a)+\left(l x_{i}-x_{i} l\right)=$ $\xi\left[x_{i}, a\right]$ and $\lambda_{a}\left(x_{i}\right)=0$ for all $i=1, \cdots, n$.

Theorem 3.5. Let $\{\cdot, \cdot\}$ be a Poisson bracket on $\mathcal{O}_{q, \delta}^{r, n}$. If $A$ is a commutative ring, $\delta_{i}=0$ for all $i=1, \ldots, n$, and $\{\cdot, \cdot\}$ is a A-bilinear function, then there exists $\xi \in A$ such that $\{a, b\}=\xi[a, b]$ for all $a, b \in \mathcal{O}_{q, \delta}^{r, n}$.

Proof. Let $a, b \in \mathcal{O}_{q, \delta}^{r, n}$ and $\xi \in A$ such that $\left\{x_{i}, X\right\}=\xi\left[x_{i}, X\right]$ for all $i=1, \ldots, n$, where $X \in \operatorname{Mon}\left\{\mathcal{O}_{q, \delta}^{r, n}\right\}, \xi$ is given by lemma (3.4). If $b=$ $\sum_{r=0}^{k} \eta_{v_{r}} x_{1}^{v_{r_{1}}} \cdots x_{n}^{v_{r_{n}}}$ since $\{\cdot, \cdot\}$ is a $A$-bilinear function and $x_{i} c=c x_{i}+$ $\delta_{i}(c)=c x_{i}$ for all $c \in A$ and $i=1, \ldots, n$, it is enough to see this when $a=x_{t_{1}}^{a_{1}} \cdots x_{t_{l}}^{a_{l}}$ with $a_{t} \neq 0$. We will show this by induction on $l$.

1) $\left(a=x_{t}^{n}\right)$ For this case we will do induction on $n$.

(a) $\left(a=x_{t}\right)$ By the above lemma we have that

$$
\begin{aligned}
\left\{x_{t}, b\right\} & =\left\{x_{t}, \sum_{r=0}^{k} \eta_{v_{r}} x_{1}^{v_{r_{1}}} \cdots x_{n}^{v_{r_{n}}}\right\}=\sum_{r=0}^{k} \eta_{v_{r}}\left\{x_{t}, x_{1}^{v_{r_{1}}} \cdots x_{n}^{v_{r_{n}}}\right\} \\
& =\sum_{r=0}^{k} \eta_{v_{r}} \xi\left[x_{t}, x_{1}^{v_{r_{1}}} \cdots x_{n}^{v_{r_{n}}}\right]=\xi \sum_{r=0}^{k} \eta_{v_{r}}\left[x_{t}, x_{1}^{v_{r_{1}}} \cdots x_{n}^{v_{r_{n}}}\right] \\
& =\xi\left[x_{t}, b\right] .
\end{aligned}
$$

(b) $\left(a=x_{t}^{n+1}\right)$

$$
\begin{aligned}
\left\{x_{t}^{n+1}, b\right\} & =\left\{x_{t}^{n}, b\right\} x_{t}+x_{t}^{n}\left\{x_{t}, b\right\}=\xi\left(\left[x_{t}^{n}, b\right] x_{t}+x_{t}^{n}\left[x_{t}, b\right]\right) \\
& =\xi\left(x_{t}^{n} b x_{t}-b x_{t}^{n+1}+x_{t}^{n+1} b-x_{t}^{n} b x_{t}\right)=\xi\left[x_{t}^{n+1}, b\right] .
\end{aligned}
$$

(c) $\left(a=x_{t}^{-1}\right)$

$$
\left\{x_{t}^{-1}, b\right\}=-x_{t}^{-1}\left\{x_{t}, b\right\} x_{t}^{-1}=\xi\left(-x_{t}^{-1}\left[x_{t}, b\right] x_{t}^{-1}\right)=\xi\left[x_{t}^{-1}, b\right] .
$$

(d) $\left(a=x_{t}^{-1-n}\right)$

$$
\begin{aligned}
\left\{x_{t}^{-1-n}, b\right\} & =\left\{x_{t}^{-n}, b\right\} x_{t}^{-1}+x_{t}^{-n}\left\{x_{t}^{-1}, b\right\} \\
& =\xi\left(\left[x_{t}^{-n}, b\right] x_{t}^{-1}+x_{t}^{-n}\left[x_{t}^{-1}, b\right]\right) \\
& =\xi\left(x_{t}^{-n} b x_{t}^{-1}-b x_{t}^{-n-1}+x_{t}^{-n-1} b-x_{t}^{-n} b x_{t}^{-1}\right) \\
& =\xi\left[x_{t}^{-n-1}, b\right] .
\end{aligned}
$$


2) $\left(a=x_{t_{1}}^{a_{1}} \cdots x_{t_{l}}^{a_{l}}\right)$

$$
\begin{aligned}
\{a, b\} & =\left\{x_{t_{1}}^{a_{1}} \cdots x_{t_{l}}^{a_{l}}, b\right\}=\left\{x_{t_{1}}^{a_{1}}, b\right\} x_{t_{2}}^{a_{2}} \cdots x_{t_{l}}^{a_{l}}+x_{t_{1}}^{a_{1}}\left\{x_{t_{2}}^{a_{2}} \cdots x_{t_{l}}^{a_{l}}, b\right\} \\
& =\xi\left(\left[x_{t_{1}}^{a_{1}}, b\right] x_{t_{2}}^{a_{2}} \cdots x_{t_{l}}^{a_{l}}+x_{t_{1}}^{a_{1}}\left[x_{t_{2}}^{a_{2}} \cdots x_{t_{l}}^{a_{l}}, b\right]\right) \\
& =\xi\left(x_{t_{1}}^{a_{1}} b x_{t_{2}}^{a_{2}} \cdots x_{t_{l}}^{a_{l}}-b a+a b-x_{t_{1}}^{a_{1}} b x_{t_{2}}^{a_{2}} \cdots x_{t_{l}}^{a_{l}}\right)=\xi[a, b] .
\end{aligned}
$$

Lemma 3.6. Let $B=\sigma(A)\left\langle x_{1}, \ldots, x_{n}\right\rangle$ be a skew PBW extension of $A$ with

1) $\sigma_{i}$ is the identity of $A$ for all $i=1, \ldots, n$.

2) $q_{i j}, a_{i j}^{(t)} \in Z(A)$ for all $1 \leqslant i, j \leqslant n$ and $t=0, \ldots, n$.

3) $\delta_{1}=0$

4) $\delta_{t}\left(q_{i j}\right)=\delta_{t}\left(a_{i j}^{(m)}\right)=0$ for all $m=0, \cdots, n$ and $i, j, t=1, \cdots, n$.

5) $p_{1 j}=0$ for all $j=1, \cdots, n$.

then there exists $\mathcal{O}_{q, \delta}^{1, n}$ with the same properties.

Proof. We will see that $B S^{-1}$ exists, showing the Ore conditions on $S$, and it is an extension of $A$ of type $\mathcal{O}_{q, \delta}^{1, n}$ for some set $S$. Let $S:=\left\{x_{1}^{m} \mid m \in \mathbb{N}\right\}$, $\mathrm{S}$ is a multiplicative set of $B$. We will see that $S$ is a right (left) Ore set.

(a) (Right) Take $P \in B$ and $s \in S$ with $s P=0$, if $P=\sum_{t \in Z} b_{t} x_{1}^{t_{1}} \cdots x_{n}^{t_{n}}$ and $s=x_{1}^{l}$ we have $0=\sum_{t \in Z} b_{t} x_{1}^{l} x_{1}^{t_{1}} \cdots x_{n}^{t_{n}}$ then $b_{t}=0$ for all $t \in Z$ then $P=0$ and $P s=0$.

(b) (Left) If $P s=0$,

$$
0=\sum_{t \in Z} b_{t} x_{1}^{t_{1}} \cdots x_{n}^{t_{n}} x_{1}^{l}=\sum_{t \in Z} b_{t} \prod_{j=2}^{n} q_{1 j}^{t_{j}} x_{1}^{t_{1}+l} x_{2}^{t_{2}} \cdots x_{n}^{t_{n}}
$$

since $q_{i j} \in A^{*}$ then $b_{t}=0$ for all $t \in Z$ and $s P=0$.

(c) (Right) Let $P=\sum_{t \in Z} b_{t} x_{1}^{t_{1}} \cdots x_{n}^{t_{n}} \in B$ and $x_{1}^{l} \in S$ then

$$
P x_{1}^{l}=\sum_{t \in Z} b_{t} \prod_{j=2}^{n} q_{1 j}^{t_{j}} x_{1}^{t_{1}+l} x_{2}^{t_{2}} \cdots x_{n}^{t_{n}}=x_{1}^{l}\left(\sum_{t \in Z} b_{t} \prod_{j=2}^{n} q_{1 j}^{t_{j}} x_{1}^{t_{1}} x_{2}^{t_{2}} \cdots x_{n}^{t_{n}}\right) \text {. }
$$

(d) (Left) Let $P=\sum_{t \in Z} b_{t} x_{1}^{t_{1}} \cdots x_{n}^{t_{n}} \in B$ and $x_{1}^{l} \in S$ then

$$
x_{1}^{l} P=\sum_{t \in Z} b_{t} x_{1}^{l} x_{1}^{t_{1}+l} x_{2}^{t_{2}} \cdots x_{n}^{t_{n}}=\left(\sum_{t \in Z} b_{t} \prod_{j=2}^{n} q_{j 1}^{t_{j}} x_{1}^{t_{1}} x_{2}^{t_{2}} \cdots x_{n}^{t_{n}}\right) x_{1}^{l} .
$$

Then $B S^{-1}$ and $S^{-1} B$ exist, so $B S^{-1} \cong S^{-1} B$. We will see that $D=B S^{-1}$ is an extension of $A$ of type $\mathcal{O}_{q, \delta}^{1, n}$ and holds the conditions. 
1) $A \hookrightarrow B \hookrightarrow D$.

2) $\frac{x_{i}}{1} \frac{a}{1}=\frac{x_{i} a}{1}=\frac{a x_{i}+\delta_{i}(a)}{1}=\frac{a x_{i}}{1}+\frac{\delta_{i}(a)}{1}=\frac{a}{1} \frac{x_{i}}{1}+\frac{\delta_{i}(a)}{1}$ for $a \in A$.

3) Let $i<j$, then

$$
\begin{aligned}
\frac{x_{j}}{1} \frac{x_{i}}{1} & =\frac{x_{j} x_{i}}{1}=\frac{q_{i j} x_{i} x_{j}+a_{i j}^{(0)}+a_{i j}^{(1)} x_{1}+\cdots+a_{i j}^{(n)} x_{n}}{1} \\
& =\frac{q_{i j}}{1} \frac{x_{i}}{1} \frac{x_{j}}{1}+\frac{a_{i j}^{(0)}}{1}+\frac{a_{i j}^{(1)}}{1} \frac{x_{1}}{1}+\cdots+\frac{a_{i j}^{(n)}}{1} \frac{x_{n}}{1} .
\end{aligned}
$$

4) Let $\frac{\sum_{t \in Z} b_{t} x_{1}^{t_{1}} \cdots x_{n}^{t_{n}}}{x_{1}^{l}} \in B$, note that

$$
\frac{1}{x_{1}} \frac{a}{1}=\frac{a}{1} \frac{1}{x_{1}} \quad\left(\frac{1}{x_{1}}\left(\frac{x_{1}}{1} \frac{a}{1}\right) \frac{1}{x_{1}}=\frac{1}{x_{1}}\left(\frac{a}{1} \frac{x_{1}}{1}\right) \frac{1}{x_{1}}\right)
$$

then

$$
\begin{aligned}
\frac{\sum_{t \in Z} b_{t} x_{1}^{t_{1}} \cdots x_{n}^{t_{n}}}{x_{1}^{l}} & =\left(\frac{x_{1}}{1}\right)^{-l} \frac{\sum_{t \in Z} b_{t} x_{1}^{t_{1}} \cdots x_{n}^{t_{n}}}{1} \\
& =\sum_{t \in Z} \frac{b_{t}}{1}\left(\frac{x_{1}}{1}\right)^{t_{1}-l} \cdots\left(\frac{x_{n}}{1}\right)^{t_{n}} .
\end{aligned}
$$

5) If $P=\sum_{t \in Z} \frac{b_{t}}{1}\left(\frac{x_{1}}{1}\right)^{t_{1}-l} \cdots\left(\frac{x_{n}}{1}\right)^{t_{n}}=\frac{0}{1}$ then $\frac{0}{1}=\left(\frac{x_{1}}{1}\right)^{l} P=$ $\frac{\sum_{t \in Z} b_{t} x_{1}^{t_{1}} \cdots x_{n}^{t_{n}}}{1}$ then there exists $x_{1}^{l} \in S$ with

$$
0=\sum_{t \in Z} b_{t} x_{1}^{t_{1}} \cdots x_{n}^{t_{n}} x_{1}^{l}=\sum_{t \in Z} b_{t} \prod_{j=2}^{n} q_{1 j}^{t_{j}} x_{1}^{t_{1}+l} \cdots x_{n}^{t_{n}}
$$

As $\prod_{j=2}^{n} q_{1 j}^{t_{j}} \in A^{*}$ then $b_{t}=0$ for all $t \in Z$ so if we denote $\frac{x_{i}}{1}:=x_{i}$, then $\operatorname{Mon}\left\{x_{1}^{ \pm 1}, x_{2}, \ldots, x_{n}\right\}$ is a $A$-basis of $D$. Note that if we put $\bar{\delta}_{i}\left(\frac{a}{1}\right)=\frac{\delta_{i}(a)}{1}$ then $\bar{\delta}_{i}\left(\frac{a_{i j}^{(t)}}{1}\right), \bar{\delta}_{i}\left(\frac{q_{i j}}{1}\right)=0$ for all $i, j=1, \ldots, n$ and $t=0, \ldots, n$, and $\bar{\delta}_{1}=0$.

Lemma 3.7. Let $\{\cdot, \cdot\}$ be a Poisson bracket over a ring $\mathrm{A}$ then $\left\{a^{l}, a^{r}\right\}=$ 0 for all $a \in A$ and $l, r \in \mathbb{N}$. 
Proof. Take $l=1$ and $r=1$ then $\{a, a\}=0$, now $\left\{a, a^{l+1}\right\}=\{a, a\} a^{l}+$ $a\left\{a, a^{l}\right\}=0$ by induction, later $\left\{a^{r}, a^{l+1}\right\}=\left\{a^{r}, a^{l}\right\} a+a^{l}\left\{a^{r}, a\right\}=$ $-a^{l}\left\{a, a^{r}\right\}=0$.

Proposition 3.8. Let $B=\sigma(A)\left\langle x_{1}, \ldots, x_{n}\right\rangle$ be a skew PBW extension of a commutative ring $A$ such that

1) $\sigma_{i}$ is the identity of $A$ for all $i=1, \ldots, n$.

2) $\delta_{i}=0$ for all $i=1, \ldots, n$.

3) For every $i$ fixed, with $i=0, \ldots, n$ and $\left(m_{1}, \ldots, m_{n}\right) \in Z \backslash\{(0, \ldots, 0\}$, $\left(1-\prod_{j=1, j \neq i}^{n} q_{i j}^{m_{j}}\right) \in A^{*}$.

4) $p_{1 j}=0$ for all $j=1, \cdots, n$.

and $\{\cdot, \cdot\}$ a Poisson bracket on $B$ then there exists a Poisson bracket $\{\cdot, \cdot\}_{0}$ on $\mathcal{O}_{q, \delta}^{1, n}$ such that $\left.\{\cdot, \cdot\}_{0}\right|_{B}=\{\cdot, \cdot\}$.

Proof. Let $D=\mathcal{O}_{q, \delta}^{1, n}$, which is given by lemma (3.6). Put $s \in B$ and define $g_{s}\left(x_{t}\right)=\left\{x_{t}, s\right\}$ for $t=1, \ldots, n, g_{s}\left(x_{1}^{-1}\right)=-x_{1}^{-1}\left\{x_{1}, s\right\} x_{1}^{-1}$, and

$$
g_{s}\left(x_{t_{1}}^{a_{1}} \cdots x_{t_{n}}^{a_{n}}\right)=g_{s}\left(x_{t_{1}}^{a_{1}}\right) x_{t_{2}}^{a_{2}} \cdots x_{t_{n}}^{a_{n}}+x_{t_{1}}^{a_{1}} g_{s}\left(x_{t_{2}}^{a_{2}} \cdots x_{t_{n}}^{a_{n}}\right) .
$$

of recurrently form.

By the universal property of basis there exists an A-homomorphism on $D$ to itself, $g_{s}$ with $\left.g_{s}\right|_{B}=\{, s\}$. We will prove that this is a derivation. Since a $g_{s}$ is an A-homomorphism then it is enough to see this to products of monomials. Let $p=x_{t_{1}}^{a_{1}} \cdots x_{t_{s}}^{a_{s}}$ and $q=x_{l_{1}}^{b_{1}} \cdots x_{l_{r}}^{b_{r}}$, we see the claimed by induction on $s$.

1) Let $s=1$, we will do induction on $r$.

(a) $(r=1)$ We will denote $p=x_{i}^{a}$ and $q=x_{j}^{b}$.

i. $(i<j)$ We have

$$
g_{s}\left(x_{i}^{a} x_{j}^{b}\right):=g_{s}\left(x_{i}^{a}\right) x_{j}^{b}+x_{i}^{a} g_{s}\left(x_{j}^{b}\right) .
$$

ii. $\left(x_{i}^{a}, x_{j}^{b} \in B\right)$ Then

$$
g_{s}\left(x_{i}^{a} x_{j}^{b}\right):=\left\{x_{i}^{a} x_{j}^{b}, s\right\}=\left\{x_{i}^{a}, s\right\} x_{j}^{b}+x_{i}^{a}\left\{x_{j}^{b}, s\right\}=g_{s}\left(x_{i}^{a}\right) x_{j}^{b}+x_{i}^{a} g_{s}\left(x_{j}^{b}\right) .
$$

iii. $\left(x_{1}^{b} \notin B, x_{j}^{a} \in B, 1 \leqslant j\right)$ Note that for all $r \in A$ we have

$$
x_{1}^{-1} r=x_{1}^{-1}\left(r x_{1}\right) x_{1}^{-1}=x_{1}^{-1}\left(x_{1} r\right) x_{1}^{-1}=r x_{1}^{-1}
$$

and

$$
x_{j} x_{1}^{-1}=x_{1}^{-1}\left(x_{1} x_{j}\right) x_{1}^{-1}=x_{1}^{-1}\left(q_{j 1} x_{j} x_{1}\right) x_{1}^{-1}=q_{j 1} x_{j} x_{1}^{-1}
$$


then for all $b \in \mathbb{Z}^{-}$and $a \in \mathbb{N}, x_{j}^{a} x_{1}^{b}=q_{j 1}^{-a b} x_{1}^{b} x_{j}^{a}=c^{-1} x_{1}^{b} x_{j}^{a}$ where $c \in Z(B)$,

$$
\begin{aligned}
c^{-1} g_{s}\left(x_{j}^{a}\right) x_{1}^{-b}+c^{-1} x_{j}^{a} g_{s}\left(x_{1}^{-b}\right) & =g_{s}\left(c^{-1} x_{j}^{a} x_{1}^{-b}\right)=g_{s}\left(x_{1}^{-b} x_{j}^{a}\right) \\
& =g_{s}\left(x_{1}^{-b}\right) x_{j}^{a}+x_{1}^{-b} g_{s}\left(x_{j}^{a}\right)
\end{aligned}
$$

and

$$
\begin{aligned}
& g_{s}\left(x_{j}^{a} x_{1}^{b}\right)=g_{s}\left(c^{-1} x_{1}^{b} x_{j}^{a}\right)=c^{-1}\left(g_{s}\left(x_{1}^{b}\right) x_{j}^{a}+x_{1}^{b} g_{s}\left(x_{j}^{a}\right)\right) \\
& =c^{-1}\left(-x_{1}^{b} g_{s}\left(x_{1}^{-b}\right) x_{1}^{b} x_{j}^{a}+x_{1}^{b} g_{s}\left(x_{j}^{a}\right)\right)=-x_{1}^{b} g_{s}\left(x_{1}^{-b}\right) c^{-1} x_{1}^{b} x_{j}^{a}+c^{-1} x_{1}^{b} g_{s}\left(x_{j}^{a}\right) \\
& =-x_{1}^{b} g_{s}\left(x_{1}^{-b}\right) x_{j}^{a} x_{1}^{b}+c^{-1} x_{1}^{b} g_{s}\left(x_{j}^{a}\right)=x_{1}^{b}\left(-g_{s}\left(x_{1}^{-b}\right) x_{j}^{a}+c^{-1} g_{s}\left(x_{j}^{a}\right) x_{1}^{-b}\right) x_{1}^{b} \\
& =x_{1}^{b}\left(x_{1}^{-b} g_{s}\left(x_{j}^{a}\right)-c^{-1} x_{j}^{a} g_{s}\left(x_{1}^{-b}\right)\right) x_{1}^{b}=g_{s}\left(x_{j}^{a}\right) x_{1}^{b}-c^{-1} x_{1}^{b} x_{j}^{a} g_{s}\left(x_{1}^{-b}\right) x_{1}^{b} \\
& =g_{s}\left(x_{j}^{a}\right) x_{1}^{b}-x_{j}^{a} x_{1}^{b} g_{s}\left(x_{1}^{-b}\right) x_{1}^{b}=g_{s}\left(x_{j}^{a}\right) x_{1}^{b}+x_{j}^{a} g_{s}\left(x_{1}^{b}\right) .
\end{aligned}
$$

(b) Let $q=x_{1}^{l_{1}} x_{2}^{l_{2}} \cdots x_{n}^{l_{n}}$ and $p=x_{1}^{b}$ then

$$
\begin{aligned}
g_{s}(p q) & =g_{s}\left(x_{1}^{l_{1}+b} x_{2}^{l_{2}} \cdots x_{n}^{l_{n}}\right):=g_{s}\left(x_{1}^{l_{1}+b}\right) x_{2}^{l_{2}} \cdots x_{n}^{l_{n}}+x_{1}^{l_{1}+b} g_{s}\left(x_{2}^{l_{2}} \cdots x_{n}^{l_{n}}\right) \\
& =\left(g_{s}\left(x_{1}^{b}\right) x_{1}^{l_{1}}+x_{1}^{b} g_{s}\left(x_{1}^{l_{1}}\right)\right) x_{2}^{l_{2}} \cdots x_{n}^{l_{n}}+x_{1}^{l_{1}+b} g_{s}\left(x_{2}^{l_{2}} \cdots x_{n}^{l_{n}}\right) \\
& =g_{s}(p) q+x_{1}^{b}\left(g_{s}\left(x_{1}^{l_{1}}\right) x_{2}^{l_{2}} \cdots x_{n}^{l_{n}}+x_{1}^{l_{1}} g_{s}\left(x_{2}^{l_{2}} \cdots x_{n}^{l_{n}}\right)\right) \\
& =g_{s}(p) q+p g_{s}(q) .
\end{aligned}
$$

2) $\left(q=x_{1}^{l_{1}} x_{2}^{l_{2}} \cdots x_{n}^{l_{n}}, p=x_{t_{1}}^{a_{1}} \cdots x_{t_{s}}^{a_{s}}\right)$. Let $T \in D$ such that $x_{2}^{t_{2}} \cdots x_{n}^{t_{n}} q=T$, later

$$
\begin{aligned}
g_{s}(p q) & =g_{s}\left(x_{t_{1}}^{a_{1}} x_{t_{2}}^{a_{2}} \cdots x_{t_{s}}^{a_{s}} q\right)=g_{s}\left(x_{t_{1}}^{a_{1}} T\right)=g_{s}\left(x_{t_{1}}^{a_{1}}\right) T+x_{t_{1}}^{a_{1}} g_{s}(T) \\
& =g_{s}\left(x_{t_{1}}^{a_{1}}\right) x_{t_{2}}^{a_{2}} \cdots x_{t_{s}}^{a_{s}} q+x_{t_{1}}^{a_{1}} g_{s}\left(x_{t_{2}}^{a_{2}} \cdots x_{t_{s}}^{a_{s}} q\right) \\
& =g_{s}\left(x_{t_{1}}^{a_{1}}\right) x_{t_{2}}^{a_{2}} \cdots x_{t_{s}}^{a_{s}} q+x_{t_{1}}^{a_{1}}\left(g_{s}\left(x_{t_{2}}^{a_{2}} \cdots x_{t_{s}}^{a_{s}}\right) q+x_{t_{2}}^{a_{2}} \cdots x_{t_{s}}^{a_{s}} g_{s}(q)\right) \\
& =\left(g_{s}\left(x_{t_{1}}^{a_{1}}\right) x_{t_{2}}^{a_{2}} \cdots x_{t_{s}}^{a_{s}}+x_{t_{1}}^{a_{1}} g_{s}\left(x_{t_{2}}^{a_{2}} \cdots x_{t_{s}}^{a_{s}}\right)\right) q+p g_{s}(q) \\
& =g_{s}(p) q+p g_{s}(q) .
\end{aligned}
$$

We will define $\{\cdot, \cdot\}_{0}$ of recurrently form on the basis of $D$. Put $p \in D$ and define

1) $f_{p}(X)=-g_{X}(p)$ for $X \in \operatorname{Mon}\left\{x_{1}, \ldots, x_{n}\right\}$.

2) $f_{p}\left(x_{1}^{-a}\right)=-x_{1}^{-a} f_{p}\left(x_{1}^{a}\right) x_{1}^{-a}, a>0$.

And for $x_{l_{1}}^{b_{1}} \cdots x_{l_{r}}^{b_{r}} \in \operatorname{Mon}\left\{x_{1}^{ \pm}, x_{2}, \ldots, x_{n}\right\}$

$$
f_{p}\left(x_{t_{1}}^{a_{1}} \cdots x_{t_{n}}^{a_{n}}\right)=f_{p}\left(x_{t_{1}}^{a_{1}}\right) x_{t_{2}}^{a_{2}} \cdots x_{t_{n}}^{a_{n}}+x_{t_{1}}^{a_{1}} f_{p}\left(x_{t_{2}}^{a_{2}} \cdots x_{t_{n}}^{a_{n}}\right) .
$$


By the universal property of the basis $f$ has a extension to an $A$-homomorphism $f$ in all $D$, now we will take $\{a, b\}_{0}:=f_{a}(b)$ and we will prove it is a bracket Poisson on $D$.

1) $\left(\{a, b\}_{0}+\{b, a\}_{0}=0\right)$ Since $\{a, b\}_{0}$ is a $A$-bilinear function, it is enough to see this when $a, b \in D$ are monomials, put $a=x_{1}^{-l} a^{\prime}$ and $b=$ $x_{1}^{-r} b^{\prime}$ where $0 \leqslant l, r, a^{\prime}=x_{1}^{a_{1}} \cdots x_{n}^{a_{n}} \in B, b^{\prime}=x_{1}^{b_{1}} \cdots x_{n}^{b_{n}} \in B$. We have

$$
\begin{aligned}
& \{a, b\}_{0}+\{b, a\}_{0}:=f_{a}(b)+f_{b}(a)=f_{a}\left(x_{1}^{-r} b^{\prime}\right)+f_{b}\left(x_{1}^{-l} a^{\prime}\right) \\
& :=\left(f_{a}\left(x_{1}^{-r}\right) b^{\prime}+x_{1}^{-r} f_{a}\left(b^{\prime}\right)\right)+\left(f_{b}\left(x_{1}^{-l}\right) a^{\prime}+x_{1}^{-l} f_{b}\left(a^{\prime}\right)\right) \\
& :=\left(-x_{1}^{-r} f_{a}\left(x_{1}^{r}\right) b+x_{1}^{-r} f_{a}\left(b^{\prime}\right)\right)+\left(-x_{1}^{-l} f_{b}\left(x_{1}^{l}\right) a+x_{1}^{-l} f_{b}\left(a^{\prime}\right)\right) \\
& :=\left(x_{1}^{-r} g_{x_{1}^{r}}(a) b-x_{1}^{-r} g_{b^{\prime}}(a)\right)+\left(x_{1}^{-l} g_{x_{1}^{l}}(b) a-x_{1}^{-l} g_{a^{\prime}}(b)\right) \\
& =\left(x_{1}^{-r} g_{x_{1}^{r}}\left(x_{1}^{-l} a^{\prime}\right) b-x_{1}^{-r} g_{b^{\prime}}\left(x_{1}^{-l} a^{\prime}\right)\right) \\
& +\left(x_{1}^{-l} g_{x_{1}^{l}}\left(x_{1}^{-r} b^{\prime}\right) a-x_{1}^{-l} g_{a^{\prime}}\left(x_{1}^{-r} b^{\prime}\right)\right) \\
& =x_{1}^{-r}\left(g_{x_{1}^{r}}\left(x_{1}^{-l}\right) a^{\prime}+x_{1}^{-l} g_{x_{1}^{r}}\left(a^{\prime}\right)\right) b-x_{1}^{-r}\left(g_{b^{\prime}}\left(x_{1}^{-l}\right) a^{\prime}+x_{1}^{-l} g_{b^{\prime}}\left(a^{\prime}\right)\right) \\
& +x_{1}^{-l}\left(g_{x_{1}^{l}}\left(x_{1}^{-r}\right) b^{\prime}+x_{1}^{-r} g_{x_{1}^{l}}\left(b^{\prime}\right)\right) a-x_{1}^{-l}\left(g_{a^{\prime}}\left(x_{1}^{-r}\right) b^{\prime}+x_{1}^{-r} g_{a^{\prime}}\left(b^{\prime}\right)\right) \\
& =x_{1}^{-r}\left(-x_{1}^{-l} g_{x_{1}^{r}}\left(x_{1}^{l}\right) x_{1}^{-l} a^{\prime}+x_{1}^{-l} g_{x_{1}^{r}}\left(a^{\prime}\right)\right) b \\
& -x_{1}^{-r}\left(-x_{1}^{-l} g_{b^{\prime}}\left(x_{1}^{l}\right) x_{1}^{-l} a^{\prime}+x_{1}^{-l} g_{b^{\prime}}\left(a^{\prime}\right)\right) \\
& +x_{1}^{-l}\left(-x_{1}^{-r} g_{x_{1}^{l}}\left(x_{1}^{r}\right) x_{1}^{-r} b^{\prime}+x_{1}^{-r} g_{x_{1}^{l}}\left(b^{\prime}\right)\right) a \\
& -x_{1}^{-l}\left(-x_{1}^{-r} g_{a^{\prime}}\left(x_{1}^{r}\right) x_{1}^{-r} b^{\prime}+x_{1}^{-r} g_{a^{\prime}}\left(b^{\prime}\right)\right) \\
& :=\left(-x_{1}^{-l-r}\left\{x_{1}^{l}, x_{1}^{r}\right\} a b+x_{1}^{-l-r}\left\{a^{\prime}, x_{1}^{r}\right\} b\right) \\
& -\left(-x_{1}^{-l-r}\left\{x_{1}^{l}, b^{\prime}\right\} a+x_{1}^{-l-r}\left\{a^{\prime}, b^{\prime}\right\}\right) \\
& +\left(-x_{1}^{-r-l}\left\{x_{1}^{r}, x_{1}^{l}\right\} b a+x_{1}^{-r-l}\left\{b^{\prime}, x_{1}^{l}\right\} a\right) \\
& -\left(-x_{1}^{-r-l}\left\{x_{1}^{r}, a^{\prime}\right\} b+x_{1}^{-r-l}\left\{b^{\prime}, a^{\prime}\right\}\right) \\
& =\left(-x_{1}^{-r-l}\left\{x_{1}^{l}, x_{1}^{r}\right\} a b-x_{1}^{-l-r}\left\{x_{1}^{r}, x_{1}^{l}\right\} b a\right) \\
& +\left(x_{1}^{-r-l}\left\{a^{\prime}, x_{1}^{r}\right\} b+x_{1}^{-r-l}\left\{x_{1}^{r}, a^{\prime}\right\} b\right) \\
& +\left(x_{1}^{-l-r}\left\{x_{1}^{l}, b^{\prime}\right\} a+x_{1}^{-r-l}\left\{b^{\prime}, x_{1}^{l}\right\} a\right) \\
& -\left(x_{1}^{-r-l}\left\{a^{\prime}, b^{\prime}\right\}+x_{1}^{-r-l}\left\{b^{\prime}, a^{\prime}\right\}\right) \\
& =\left(-x_{1}^{-r-l}\left\{x_{1}^{l}, x_{1}^{r}\right\} a b+x_{1}^{-l-r}\left\{x_{1}^{r}, x_{1}^{l}\right\} b a\right)=0 \text {. }
\end{aligned}
$$

2) $\left(\{a, a\}_{0}=0\right)$ Let $a=x_{1}^{l} a^{\prime}$ where $a^{\prime}=\sum_{t} \eta_{t} x_{1}^{t_{1}} \cdots x_{n}^{t_{n}} \in B$ and $l \leqslant 0$ then

$$
\{a, a\}_{0}:=f_{a}\left(x_{1}^{l} a^{\prime}\right)=\sum_{t} \eta_{t} f_{a}\left(x_{1}^{l}\left(x_{1}^{t_{1}} \cdots x_{n}^{t_{n}}\right)\right)
$$




$$
\begin{aligned}
& :=\sum_{t} \eta_{t}\left(f_{a}\left(x_{1}^{l}\right) x_{1}^{t_{1}} \cdots x_{n}^{t_{n}}+x_{1}^{l} f_{a}\left(x_{1}^{t_{1}} \cdots x_{n}^{t_{n}}\right)\right) \\
& =f_{a}\left(x_{1}^{l}\right) a^{\prime}+x_{1}^{l} f_{a}\left(a^{\prime}\right):=-x_{1}^{l} f_{a}\left(x_{1}^{-l}\right) x_{1}^{l} a^{\prime}+x_{1}^{l} f_{a}\left(a^{\prime}\right) \\
& =x_{1}^{l} g_{x_{1}^{-l}}\left(x_{1}^{l} a^{\prime}\right) x_{1}^{l} a^{\prime}-x_{1}^{l} g_{a^{\prime}}\left(x_{1}^{l} a^{\prime}\right) \\
& =x_{1}^{l}\left(g_{x_{1}^{-l}}\left(x_{1}^{l}\right) a^{\prime}+x_{1}^{l} g_{x_{1}^{-l}}\left(a^{\prime}\right)\right) x_{1}^{l} a^{\prime}-x_{1}^{l}\left(g_{a^{\prime}}\left(x_{1}^{l}\right) a^{\prime}+x_{1}^{l} g_{a^{\prime}}\left(a^{\prime}\right)\right) \\
& =x_{1}^{l} g_{x_{1}^{-l}}\left(x_{1}^{l}\right) a^{\prime} a+x_{1}^{2 l} g_{x_{1}^{-l}}\left(a^{\prime}\right) x_{1}^{l} a^{\prime}-x_{1}^{l} g_{a^{\prime}}\left(x_{1}^{l}\right) a^{\prime}-x_{1}^{2 l} g_{a^{\prime}}\left(a^{\prime}\right) \\
& =\left(x_{1}^{l} g_{x_{1}^{-l}}\left(x_{1}^{l}\right) a^{\prime} a-x_{1}^{2 l} g_{a^{\prime}}\left(a^{\prime}\right)\right)+\left(x_{1}^{2 l} g_{x_{1}^{-l}}\left(a^{\prime}\right) x_{1}^{l} a^{\prime}-x_{1}^{l} g_{a^{\prime}}\left(x_{1}^{l}\right) a^{\prime}\right) \\
& =\left(x_{1}^{2 l} g_{x_{1}^{-l}}\left(x_{1}^{-l}\right) x_{1}^{l} a^{\prime} a-x_{1}^{2 l} g_{a^{\prime}}\left(a^{\prime}\right)\right)+\left(x_{1}^{2 l} g_{x_{1}^{-l}}\left(a^{\prime}\right) x_{1}^{l} a^{\prime}+x_{1}^{2 l} g_{a^{\prime}}\left(x_{1}^{-l}\right) x_{1}^{l} a^{\prime}\right) \\
& :=x_{1}^{2 l}\left(\left\{x_{1}^{-l}, x_{1}^{-l}\right\} a a-\left\{a^{\prime}, a^{\prime}\right\}\right)+x_{1}^{2 l}\left(\left\{a^{\prime}, x_{1}^{-l}\right\}+\left\{x_{1}^{-l}, a^{\prime}\right\}\right) a=0 .
\end{aligned}
$$

3) $\left(\{a b, c\}_{0}=\{a, c\}_{0} b+a\{b, c\}_{0}\right)$ It can be proved the same way we proved that $g_{X}(\cdot)$ is a derivations for all $X \in \operatorname{Mon}\left\{x_{1}, \ldots, x_{n}\right\}$.

Theorem 3.9. Let $B=\sigma(A)\left\langle x_{1}, \ldots, x_{n}\right\rangle$ be a skew PBW extension of $A$ as above and $\{\cdot, \cdot\}$ a poisson bracket on $B$, then there exists $\xi \in A$ such that $\{a, b\}=\xi[a, b]$ for all $a, b \in B$.

Proof. Since $\{\cdot, \cdot\}$ is a Poisson bracket on $B$ then there exists a poisson bracket $\{\cdot, \cdot\}_{0}$ on $\mathcal{O}_{q, \delta}^{1, n}$ with $\left.\{\cdot, \cdot\}_{0}\right|_{B}=\{\cdot, \cdot\}$ which is A-bilinear function, by theorem (3.5) there exists $\xi \in A$ such that $\{a, b\}_{0}=\xi[a, b]$ for all $a, b \in \mathcal{O}_{q, \delta}^{1, n}$ so if $a, b \in B$ then $\{a, b\}=\{a, b\}_{0}=\xi[a, b]$.

\section{Some examples}

In this section we will show some algebras where we can give a characterization of the poisson brackets.

1) The algebra of $q$-differentiable operators. $D_{q, h}[x, y]$. Let $q, h \in \mathbb{K}$, $q \neq 0$ consider $\mathbb{K}[y][x ; \sigma, \delta], \sigma(y):=q y$ and $\delta(y):=h$. By definitions of skew polynomial ring, it is the $\mathbb{K}$-algebra defined by the relation $x y=q t x+h$. If we put $h=0$ and $q^{l}-1 \in \mathbb{K}^{*}$ for all $l \in \mathbb{N}$ we have that $x r=r x$ and $y r=r y$ for all $r \in \mathbb{K}$.

2) The algebra of linear partial q-dilatation operators. For a fixed $q \in \mathbb{K}-\{0\}$, the $\mathbb{K}$-algebra of linear partial q-dilatation operators with polynomial coefficients, respectively, with rational coefficients, is $\mathbb{K}\left[t_{1}, \ldots, t_{n}\right]\left[H_{1}^{(q)}, \ldots, H_{m}^{(q)}\right]$, respectively $\mathbb{K}\left(t_{1}, \ldots, t_{n}\right)\left[H_{1}^{(q)}, \ldots, H_{m}^{(q)}\right]$ $n \leqslant m$, subject to the relations:

$$
t_{j} t_{i}=t_{i} t_{j}, \quad 1 \leqslant i<j \leqslant n,
$$




$$
\begin{array}{rc}
H_{i}^{(q)} t_{i}=q t_{i} H_{i}^{(q)}, & 1 \leqslant i \leqslant n, \\
H_{j}^{(q)} t_{i}=t_{i} H_{j}^{(q)}, & i \neq j, \\
H_{i}^{(q)} H_{j}^{(q)}=H_{j}^{(q)} H_{i}^{(q)}, & 1 \leqslant i \leqslant n .
\end{array}
$$

If we take $n=m=1$ and $q^{l}-1 \in \mathbb{K}^{*}$ for all $l \in \mathbb{N}$.

3) Multiplicative analogue of the Weyl algebra. The $\mathbb{K}$-algebra $O_{n}\left(\lambda_{i j}\right)$ is generated by $x_{1}, \ldots, x_{n}$ subject to the relations:

$$
x_{j} x_{i}=\lambda_{i j} x_{i} x_{j}, \quad 1 \leqslant i<j \leqslant n,
$$

where $\lambda_{i j} \in \mathbb{K}-\{0\}$. If we take $n>1$ and $\lambda_{i j}$ as $\mathbb{N}$ independent, i.e. for all $i=1, \ldots, n$ and $m \in \mathbb{N}^{n}-\left\{(0, \ldots, 0), 1-\prod_{j \neq i} \lambda_{i j}^{m_{j}} \in \mathbb{K}^{*}\right.$.

4) 3-dimensional skew polynomial algebra $A$. It is given by the relations

$$
y z-\alpha z y=\lambda, \quad z x-\beta x z=\mu, \quad x y-\gamma y x=v
$$

such that $\lambda, \mu, v \in \mathbb{K}+\mathbb{K} x+\mathbb{K} y+\mathbb{K} z$, and $\alpha, \beta, \gamma \in \mathbb{K}-\{0\}$. If we take $\lambda, \mu=0$ and $\alpha, \beta, \gamma, \mathbb{N}$-independent.

5) Quantum Space $S_{q}$. Let $\mathbb{K}$ be a commutative ring and let $\mathbf{q}=\left[q_{i j}\right]$ be a matrix with entries in $\mathbb{K}^{*}$ such that $q_{i i}=1=q_{i j} q_{j i}$ for all $1 \leqslant i, j \leqslant n$. The $\mathbb{K}$-algebra $S_{q}$ is generated by $x_{1}, \ldots, x_{n}$, subject to the relations

$$
x_{i} x_{j}=q_{i j} x_{j} x_{i} .
$$

If we take $n>1$ and $q_{i j} \mathbb{N}$-independent.

6) Witten's deformation of $\mathcal{U}(\mathcal{S} \mathcal{L}(2, \mathbb{K}))$. Let $\underline{\xi}=\left(\xi_{1}, \ldots, \xi_{7}\right)$ a 7 -tuple of parameters, it is generated by $x, y, z$ subject to relations

$$
x z-\xi_{1} z x=\xi_{2} x, \quad z y-\xi_{3} y z=\xi_{4} y, \quad y x-\xi_{5} x y=\xi_{6} z^{2}+\xi_{7} z .
$$

If we take $\xi_{7}, \xi_{6}, \xi_{2}=0$ and $\xi_{1}, \xi_{3}, \xi_{5} \in \mathbb{K}^{*}$ and $\mathbb{N}$-independent.

7) Quantum symplectic space. $O_{q}\left(\mathcal{S P}\left(\mathbb{K}^{2 n}\right)\right)$. For every nonzero element $q \in \mathbb{K}$, one defines this quantum algebra $O_{q}\left(\mathcal{S P}\left(\mathbb{K}^{2 n}\right)\right)$ to be the algebra generated by $\mathbb{K}$ and the variables $y_{1} \cdots, y_{n}, x_{1}, \ldots, x_{n}$ subject to the relations

$$
\begin{gathered}
y_{j} x_{i}=q^{-1} x_{i} y_{j}, \quad y_{i} y_{j}=y_{i} y_{j}, \quad 1 \leqslant i<j \leqslant n, \\
x_{j} x_{i}=q^{-1} x_{i} x j, \quad x_{j} y_{i}=q y_{i} x_{j}, \quad 1 \leqslant i<j \leqslant n, \\
x_{i} y_{i}-q^{2} y_{i} x_{i}=\left(q^{2}-1\right) \sum_{l=1}^{i-1} q^{i-l} y_{l} x_{l}, \quad 1 \leqslant i \leqslant n .
\end{gathered}
$$

If we take $n=1$ and $q \mathbb{N}$-independent. 


\section{Acknowledgements}

The author would like to thank Jose Oswaldo Lezama Serrano for many helpful suggestions which were so important to develop the article.

\section{References}

[1] V.A Artamonov. Action of Hopf algebras on General Quantum Malcev power series and Quantum planes, J. Math. Sci., 2006, 134, N 1, pp. 1773-1798.

[2] Gallego, C. and Lezama, O., Gröbner bases for ideals of sigma-PBW extensions, Communications in Algebra, 39 (1), 2011, 50-75.

[3] Reyes, M. A., Ring and Module Theoretic Properties of -PBW Extensions, Ph.D. Thesis, Universidad Nacional de Colombia, 2013.

[4] O. Lezama, J. P. Acosta and A. Reyes, Prime ideals of skew PBW extensions, Rev. Un. Mat. Argentina, vol. 56, no. 2, pp. 39-55, 2015.

[5] O. Lezama and A. Reyes, Some Homological Properties of Skew PBW Extensions, Comm. Algebra, vol. 42, pp. 1200-1230, 2014.

[6] A. Reyes and H. Suárez, Armendariz property for skew PBW extensions and their classical ring of quotients, Rev. Integr. Temas Mat., vol. 34, no. 2, pp. 147-168, 2016 .

[7] Suarez,H, Kozulity for graded skew PBW extensions, Comm. in Algebra, 45(10), 2017, 4569-4580.

[8] Acosta, J.P., Chaparro, C., Lezama, O., Ojeda, I., and Venegas, C., Ore and Goldie theorems for skew PBW extensions, Asian-European Journal of Mathematics, 6(4), 2013, 1350061-1-1350061-20.

\section{CONTACT INFORMATION}

Brian Andres

Zambrano Luna
Seminario de Álgebra Constructiva - $S A C^{2}$

Departamento de Matemáticas, Universidad

Nacional de Colombia, Sede Bogotá

E-Mail(s): bazambranol@unal.edu.co

Received by the editors: 16.02.2018. 